\title{
Phonon dispersions and piezoelectricity in bulk and multilayers of hexagonal boron nitride
}

\author{
K. H. Michel and B. Verberck \\ Departement Fysica, Universiteit Antwerpen, Groenenborgerlaan 171, B-2020 Antwerpen, Belgium \\ (Received 5 November 2010; revised manuscript received 31 January 2011; published 24 March 2011)
}

\begin{abstract}
A unified theory of phonon dispersions and piezoelectricity in bulk and multilayers of hexagonal boron nitride (h-BN) is derived. The dynamical matrix is calculated on the basis of an empirical force constant model of intralayer valence and interlayer van der Waals interactions. Coulomb interactions are calculated by Ewald's method, adapted for the three-dimensional (3D) and the multilayer case. The deformation of the ionic charge distribution with long-wave lattice displacements is taken into account. Special attention is devoted to the nonanalytic long-range Coulomb contribution to the dynamical matrix which is different for the 3D crystal and the multilayer case. Consequently there is a splitting of the transverse optical (TO) and longitudinal optical (LO) phonon branches of $E_{1 u}$ symmetry and a discontinuity of the $A_{2 u}$ branch at the $\Gamma$ point in 3D h-BN. No such splitting and discontinuity at $\Gamma$ are present in multilayer crystals with a finite number $\mathcal{N}$ of layers. There a diverging bundle of $\mathcal{N}$ overbending optical phonon branches emerges from $\Gamma$. Born's long-wave theory is applied and extended for the study of piezoelectricity in layered crystals. While 3D h-BN and h-BN multilayers with an even number of layers (symmetry $D_{6 h}$ ) are not piezoelectric, multilayers with an uneven number of $\mathcal{N}_{u}$ layers (symmetry $D_{3 h}$ ) are piezoelectric; the piezoelectric coefficient $e_{1,11}$ is inversely proportional to $\mathcal{N}_{u}$.
\end{abstract}

DOI: 10.1103/PhysRevB.83.115328 PACS number(s): 63.22.Np, 77.65.-j, 61.46.-w, 68.65.Ac

\section{INTRODUCTION}

The experimental discovery of graphene and other freestanding two-dimensional (2D) crystals ${ }^{1-3}$ has opened the path for the synthesis of ultrathin sheets of crystalline material with novel physical properties. Most prominent is graphene-a monoatomic layer of crystalline $\mathrm{C}$ with hexagonal structureobtained by micromechanical cleavage of graphite as threedimensional (3D) layered parent material. Likewise 2D hexagonal boron nitride ( $\mathrm{h}-\mathrm{BN}$ ) is produced from $3 \mathrm{D}$ h-BN. Recently a single layer of $\mathrm{BN}$ has been fabricated by controlled energetic electron irradiation-induced layer-by-layer sputtering. ${ }^{4}$ Both materials differ in their electronic properties and in their chemical bonding. While graphene is a conductor, 2D h-BN is a true insulator; ${ }^{1}$ 3D h-BN has a direct band gap in the ultraviolet region. ${ }^{5}$ Graphene is purely covalent; $2 \mathrm{D} \mathrm{h}$-BN built from III-V elements is a dielectric with partially covalent and ionic bonds. ${ }^{6}$ Both graphene and $2 \mathrm{D}$ h-BN can be regarded as building materials for one-dimensional nanotubes (for a review see Refs. 7-9).

In the following we will turn our attention to the lattice dynamics of 3D h-BN and of h-BN multilayers. The synthesis of both on-substrate and free-standing few-atomic-layer sheets of h-BN has been achieved by micromechanical cleavage ${ }^{10}$ and a chemical-solution-derived method. ${ }^{11}$ Recently, progress in the large-scale fabrication of $\mathrm{BN}$ nanosheets has been reported. ${ }^{12}$ In early work, normal modes at the $\Gamma$ point of the Brillouin zone have been measured in $3 \mathrm{D}$ h-BN by infrared ${ }^{13}$ and first-order Raman scattering ${ }^{13-15}$ techniques. Phonon spectra have been obtained by high-resolution electron energy loss spectroscopy of an epitaxial monolayer film of h-BN on $\mathrm{Ni}(111) .{ }^{16,17}$ Theoretical work on $\mathrm{h}-\mathrm{BN}$ has been motivated by the fabrication of BN nanotubes. ${ }^{18,19}$ Phonon dispersions of 3D $\mathrm{h}-\mathrm{BN}^{20-22}$ and of h-BN monolayers ${ }^{23-26}$ have been calculated by first-principles and tight-binding methods. Second-order Raman scattering has been used to probe phonon dispersions away from the $\Gamma$ point. $^{27}$ Recently, inelastic $\mathrm{x}$-ray scatter- ing (IXS) experiments have been performed on 3D h-BN. Besides the elastic constants, ${ }^{28}$ phonon dispersions have been measured and compared with ab initio calculations. ${ }^{29}$ The present authors have studied the phonon dispersions as well as elastic and piezoelectric properties of $2 \mathrm{D}$ h-BN by analytical methods. ${ }^{30}$

While present-day theory is based most often on ab initio calculations, the analytical approach provides additional theoretical insight and is complementary to the former. In particular, due to the presence of long-range Coulomb interactions the lattice dynamics of ionic crystals depends on the dimensionality of the crystal. ${ }^{24,31}$ The analytical methods allow us to separate the different types of interactions (Coulomb, covalent, and van der Waals) and to treat 3D h-BN, a single layer (2D h-BN), and a slab of an arbitrary large number of layers in a unified way.

The content of the paper is as follows. First (Sec. II) we set up the dynamical matrix of $3 \mathrm{D}$ h-BN, taking into account covalent, Coulomb, and van der Waals interactions. The phonon dispersion relations are calculated in Sec. III. The splitting of the highest optical phonon branches at the $\Gamma$ point is derived by numerical and analytical calculations. Next (Sec. IV) we study systems of h-BN multilayers. In constructing the dynamical matrix we use 2D Fourier transforms for the in-plane components of the interactions and summations in direct space for out-of-plane components. Solution of the secular equation gives the phonon dispersion relations. The evolution of the highest optical branches with increasing layer number $\mathcal{N}$ is investigated. Next (Sec. V) we study by analytical methods the overbending of the highest optical phonon branch as a function of the layer number $\mathcal{N}$. In Sec. VI we use Born's long-wave method ${ }^{31,32}$ to study piezoelectricity of multilayer systems. Symmetry implies that piezoelectricity is absent in 3D h-BN and in h-BN slabs with an even number of layers. In slabs with an uneven number $\mathcal{N}$ of layers, the piezoelectric stress coefficient is different from zero 


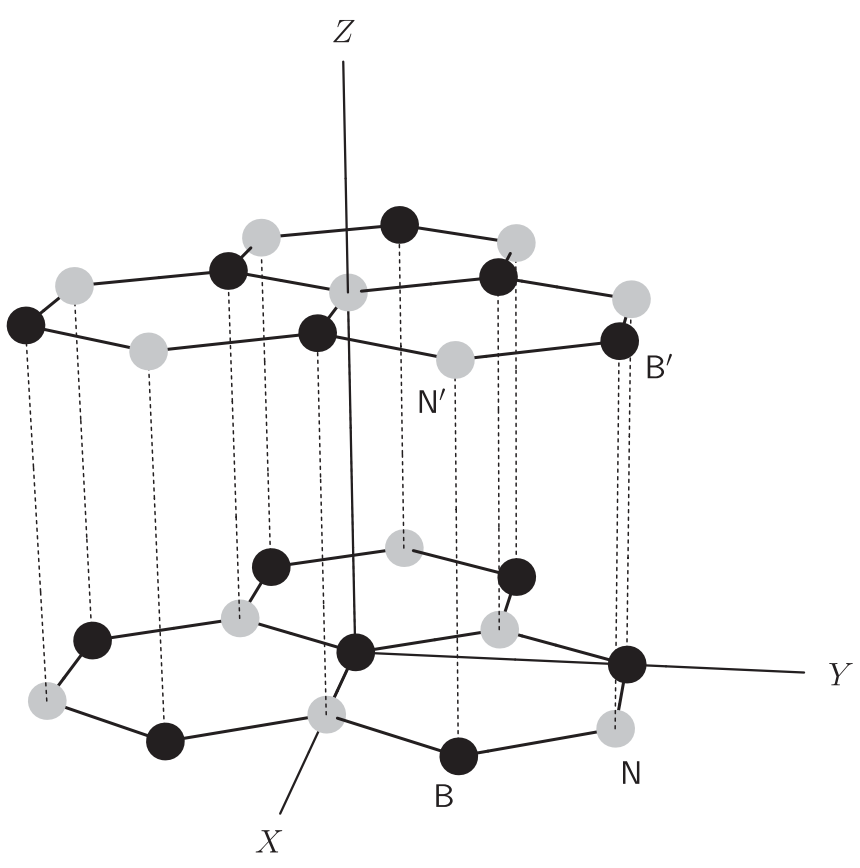

FIG. 1. Excerpt of two neighboring h-BN planes.

and decreases as $1 / \mathcal{N}$. Concluding remarks (Sec. VII) close the paper.

\section{ELEMENTS OF LATTICE DYNAMICS}

We recall some basic concepts of lattice dynamics to be applied to 3D h-BN. The space group is $D_{6 h}^{4}\left(\mathrm{PG}_{3} / \mathrm{mmc}\right)$. The crystal is built up from a superposition of (002) h-BN atomic planes. ${ }^{33}$ The planes are at distance $c / 2$; each $\mathrm{B}$ atom is on top of an $\mathrm{N}$ atom in the adjacent plane and vice versa (Fig. 1). The primitive lattice vectors, expressed in Cartesian coordinates, are $\vec{a}^{(1)}=(\sqrt{3} a / 2, a / 2,0), \vec{a}^{(2)}=(\sqrt{3} a / 2,-a / 2,0), \vec{a}^{(3)}=$ $(0,0, c)$. The volume of the unit cell is $v_{3 \mathrm{D}}=a^{2} c \sqrt{3 / 2}$. Here $a=\sqrt{3} a_{\mathrm{BN}}$, where $a_{\mathrm{BN}}=1.446 \AA$ is the bond length distance between nearest neighbor $\mathrm{B}$ and $\mathrm{N}$ atoms in a h-BN plane; the distance between equivalent planes is $c=6.660 \AA$. $\mathrm{Ab}$ initio calculations show that the layer stacking is determined by electrostatic forces while the van der Waals forces fix the interlayer distance. ${ }^{34}$ Each unit cell of the crystal contains four atoms to be labeled by a superscript $\kappa \in\left\{\mathrm{B}, \mathrm{N}, \mathrm{B}^{\prime}, \mathrm{N}^{\prime}\right\}$ and located at positions $\vec{r}^{\kappa}=(0,0,0),(a / \sqrt{3}, 0,0)$ for $\kappa=\mathrm{B}$, $\mathrm{N}$, respectively, and $\vec{r}^{\kappa}=(a / \sqrt{3}, 0, c / 2),(0,0, c / 2)$ for $\kappa=$ $\mathrm{B}^{\prime}, \mathrm{N}^{\prime}$, respectively. Here we use the prime as an index to distinguish between nearest neighbor atoms in adjacent planes. The equilibrium position in the crystal of the $\kappa$ th atom in the $\vec{n}$ th unit cell is given by

$$
\vec{X}(\vec{n} \kappa)=\vec{X}(\vec{n})+\vec{r}^{\kappa} .
$$

Here $\vec{X}(\vec{n})=n_{1} \vec{a}^{(1)}+n_{2} \vec{a}^{(2)}+n_{3} \vec{a}^{(3)}$ is a lattice vector; $\vec{n}=\left(n_{1}, n_{2}, n_{3}\right)$ is a triplet of integers which specifies the position of the unit cell. The Brillouin zone (BZ) of $\Gamma_{h}$ is shown in Fig. 2. The basis reciprocal lattice vectors are $\vec{b}^{(1)}=2 \pi(1 / \sqrt{3} a, 1 / a, 0), \quad \vec{b}^{(2)}=2 \pi(1 / \sqrt{3} a,-1 / a, 0)$, $\vec{b}^{(3)}=2 \pi(0,0,1 / c)$.

The basic theoretical quantity in lattice dynamics is the dynamical matrix $D(\vec{q})$, which is a function of the wave

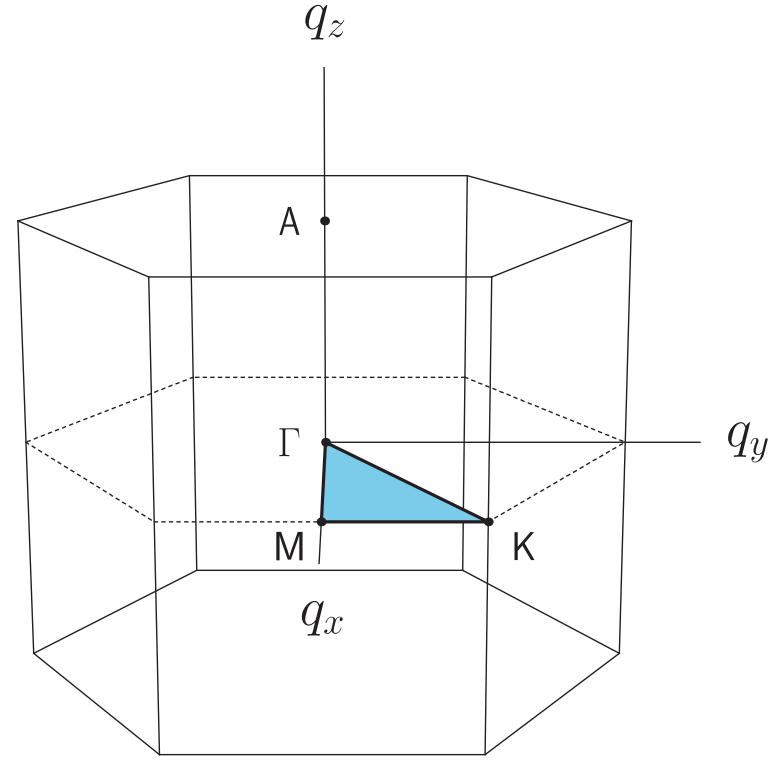

FIG. 2. (Color online) Brillouin zone of 3D h-BN; plane containing $\Gamma M K$ is $\mathrm{BZ}$ of $2 \mathrm{D} \mathrm{h}-\mathrm{BN}$.

vector $\vec{q}=\left(q_{x}, q_{y}, q_{z}\right)$. In the case of $3 \mathrm{D}$ h-BN, $D(\vec{q})$ is of dimension $12 \times 12$ with elements $D_{i j}^{\kappa \kappa^{\prime}}(\vec{q}) ; i(j)$ labels the $x$, $y, z$ components of the atomic displacements. Since $\mathrm{h}-\mathrm{BN}$ is a crystal with partial covalent and partial ionic bond character, we write $D$ as a sum of a covalent term $F$, a Coulomb term $C$, and a van der Waals term $J$ :

$$
D(\vec{q})=F\left(\vec{q}_{\perp}\right)+C(\vec{q})+J(\vec{q}) .
$$

The covalent term, parametrized in terms of a model of in-plane force constants $\left\{f\left(\vec{n}_{\perp} \kappa ; \vec{n}_{\perp}^{\prime} \kappa^{\prime}\right)\right\}$, has the matrix elements

$$
\begin{aligned}
F_{i j}^{\kappa \kappa^{\prime}}\left(\vec{q}_{\perp}\right)= & -\frac{1}{\sqrt{m_{\kappa} m_{\kappa^{\prime}}}}\left[\sum_{\vec{n}_{\perp}^{\prime}} f_{i j}\left(\overrightarrow{0}_{\perp} \kappa ; \vec{n}_{\perp}^{\prime} \kappa^{\prime}\right)\right. \\
& \left.\times e^{i \vec{q}_{\perp} \cdot\left[\vec{X}\left(\vec{n}_{\perp}^{\prime} \kappa^{\prime}\right)-\vec{X}\left(\overrightarrow{0}_{\perp} \kappa\right)\right]}-\delta_{\kappa \kappa^{\prime}} \sum_{\vec{n}_{\perp}^{\prime} \kappa^{\prime \prime}} f_{i j}\left(\overrightarrow{0}_{\perp} \kappa ; \vec{n}_{\perp}^{\prime} \kappa^{\prime \prime}\right)\right] .
\end{aligned}
$$

Here $\vec{n}_{\perp}=\left(n_{1}, n_{2}\right) ; \vec{n}_{\perp}$ and $\vec{n}_{\perp}^{\prime}$ refer to a same plane, and $\vec{q}_{\perp}=\left(q_{x}, q_{y}\right)$. The prime on the summation signs in the last equation indicates that the term with $(\overrightarrow{0} \kappa)=\left(\vec{n}_{\perp}^{\prime} \kappa^{\prime}\right)$ is excluded. Additional details on the force constant model will be given in Sec. III.

The van der Waals term accounts for the coupling between atoms in nearest neighbor planes due to London dispersion forces. The corresponding force constants $\left\{h_{i j}\left(\vec{n} \kappa ; \vec{n}^{\prime} \kappa^{\prime}\right)\right\}$ with $\left(\vec{n} \kappa \neq \vec{n}^{\prime} \kappa^{\prime}\right)$ are calculated by means of an atom-atom van der Waals potential. The matrix elements of $J(\vec{q})$ read

$$
\begin{aligned}
J_{i j}^{\kappa \kappa^{\prime}}(\vec{q})= & -\frac{1}{\sqrt{m_{\kappa} m_{\kappa^{\prime}}}}\left[\sum_{\vec{n}^{\prime}} h_{i j}\left(\overrightarrow{0} \kappa ; \vec{n}^{\prime} \kappa^{\prime}\right)\right. \\
& \left.\times e^{i \vec{q} \cdot\left[\vec{X}\left(\vec{n}^{\prime} \kappa^{\prime}\right)-\vec{X}(\overrightarrow{0} \kappa)\right]}-\delta_{\kappa \kappa^{\prime}} \sum_{\vec{n}^{\prime} \kappa^{\prime \prime}} h_{i j}\left(\overrightarrow{0} \kappa ; \vec{n}^{\prime} \kappa^{\prime \prime}\right)\right] .
\end{aligned}
$$

Details on the van der Waals potential will be given in Sec. III. 
The Coulomb part is calculated by means of the Ewald method. ${ }^{31,32}$ The elements of $C(\vec{q})$ are

$$
C_{i j}^{\kappa \kappa^{\prime}}(\vec{q})=\frac{1}{\sqrt{m_{\kappa} m_{\kappa^{\prime}}}} \Phi_{i j}^{\kappa \kappa^{\prime}}(\vec{q})-\delta_{\kappa \kappa^{\prime}} \frac{1}{m_{\kappa}} \sum_{\kappa^{\prime \prime}} \Phi_{i j}^{\kappa \kappa^{\prime \prime}}(\vec{q}=\overrightarrow{0}),
$$

with coupling parameters

$$
\Phi_{i j}^{\kappa \kappa^{\prime}}(\vec{q})=\left.\Phi_{i j}^{\kappa \kappa^{\prime}}(\vec{q})\right|_{1}+\left.\Phi_{i j}^{\kappa \kappa^{\prime}}(\vec{q})\right|_{2} .
$$

Here $\left.\Phi\right|_{1}$ and $\left.\Phi\right|_{2}$ are given by summations over the reciprocal and the direct lattice, respectively. One has for a 3D ionic crystal the expression

$$
\begin{aligned}
\left.\Phi_{i j}^{\kappa \kappa^{\prime}}(\vec{q})\right|_{1}= & \frac{4 \pi}{v_{3 \mathrm{D}}} e_{\kappa} e_{\kappa^{\prime}} \sum_{\vec{\tau}} \frac{(\vec{\tau}-\vec{q})_{i}(\vec{\tau}-\vec{q})_{j}}{|\vec{\tau}-\vec{q}|^{2}} \\
& \times \exp \left(-\frac{|\vec{\tau}-\vec{q}|^{2}}{4 \eta}\right) e^{\left.i \vec{\tau} \cdot \vec{r}\left(\kappa^{\prime}\right)-\vec{r}(\kappa)\right]},
\end{aligned}
$$

where $\vec{\tau}$ is a reciprocal lattice vector, $\eta$ is the range parameter of the Gaussian charge distribution in real space, and $e_{\kappa}$ is the effective charge of atom $\kappa$. [Here and in the following we continue to use the term atom for charged atoms (ions).] The second term on the right-hand side (rhs) of Eq. (6) reads

$$
\begin{aligned}
\left.\Phi_{i j}^{\kappa \kappa^{\prime}}(\vec{q})\right|_{2}= & -e_{\kappa} e_{\kappa^{\prime}} \eta^{\frac{3}{2}} \sum_{\vec{n}^{\prime}} H_{i j}^{\kappa \kappa^{\prime}}\left(\sqrt{\eta} \mid \vec{X}\left(\vec{n}^{\prime} \kappa^{\prime}\right)\right. \\
& -\vec{X}(\overrightarrow{0} \kappa) \mid) e^{i \vec{q} \cdot\left[\vec{X}\left(\vec{n}^{\prime} \kappa^{\prime}\right)-\vec{X}(\overrightarrow{0} \kappa)\right]},
\end{aligned}
$$

where, writing $x$ for $|\vec{x}|=\sqrt{x_{1}^{2}+x_{2}^{2}+x_{3}^{2}}$,

$$
\begin{aligned}
H_{i j}^{\kappa \kappa^{\prime}}(\vec{x})= & \frac{x_{i} x_{j}}{x^{2}}\left[\frac{3}{x^{3}} \operatorname{erfc}(x)+\frac{2}{\sqrt{\pi}}\left(\frac{3}{x^{2}}+2\right) e^{-x^{2}}\right] \\
& -\delta_{i j}\left[\frac{1}{x^{3}} \operatorname{erfc}(x)+\frac{2}{\sqrt{\pi}} \frac{1}{x^{2}} e^{-x^{2}}\right] .
\end{aligned}
$$

The divergent term arising in Eq. (8a) for $\left(\vec{n}^{\prime} \kappa^{\prime}\right)=(\overrightarrow{0} \kappa)$ is compensated by the same term from $\Phi_{i j}^{\kappa \kappa^{\prime}}(\vec{q}=\overrightarrow{0})$ in Eq. (5).

The term with $\vec{\tau}=\overrightarrow{0}$ on the rhs of Eq. (7) yields the contribution

$$
\left.C_{i j}^{\kappa \kappa^{\prime}}(\vec{q}, \vec{\tau}=\overrightarrow{0})\right|_{1}=\frac{4 \pi e_{\kappa} e_{\kappa^{\prime}}}{v_{3 \mathrm{D}} \sqrt{m_{\kappa} m_{\kappa^{\prime}}}} \frac{q_{i} q_{j}}{|\vec{q}|^{2}} e^{-\frac{q^{2}}{4 \eta}}
$$

to the dynamical matrix. Here $|\vec{q}|=\sqrt{q_{x}^{2}+q_{y}^{2}+q_{z}^{2}}$. Expression (9) becomes nonregular at $\vec{q}=\overrightarrow{0}$, since its limit value depends on the direction of $\vec{q} \rightarrow \overrightarrow{0}$. This feature reflects the sample shape dependence of the long-range Coulomb forces in three dimensions. ${ }^{35}$ The nonregular term plays the role of a macroscopic electric field ${ }^{31,32}$ in the equations of motion of the crystal. As was first discovered in cubic ionic crystals this symmetry-breaking field leads to the well-known splitting between LO (longitudinal optical) and TO (transverse optical) phonons at the $\Gamma$ point of the BZ. ${ }^{36}$

Before calculating the phonon dispersion relations, a specification of the ionic charges is in order. By means of density functional theory one estimates that for a free-standing h-BN sheet 0.56 electrons are transferred from B to N. ${ }^{37} \mathrm{We}$ will take effective charges $e_{\mathrm{B}}^{\perp}=-e_{\mathrm{N}}^{\perp}=0.56 e$ for in-plane displacements (perpendicular to the $\vec{c}$ axis), and $e_{\mathrm{B}}^{\|}=-e_{\mathrm{N}}^{\|}=$ $0.186 e$ for out-of-plane displacements (parallel to $\vec{c}$ ), where $e$ is the elementary charge. The ratio $e_{\mathrm{B}}^{\perp} / e_{\mathrm{B}}^{\|}$is close to the result of ab initio calculations. ${ }^{21}$ We will use these values of ionic charges for the calculation of the short-range (regular) Coulomb terms of the dynamical matrix. The range parameter $\eta$ will be given the value $\eta=10 / a^{2}$, i.e., $3.33 / a_{\mathrm{BN}}^{2}$. In order to deal with the nonregular Coulomb contribution to the dynamical matrix, we have to take into account the deformation of the ions due to the macroscopic electric field which is effective at long-wavelength displacements. Hence we will use Eq. (9) with larger values of the effective charges: ${ }^{39}$ $e_{\mathrm{B}}^{* \perp}=-e_{\mathrm{N}}^{* \perp}=1.218 e, e_{\mathrm{B}}^{* \|}=-e_{\mathrm{N}}^{* \|}=0.405 e$. These values agree with those of the ab initio calculations ${ }^{21}$ by identifying $e_{\mathrm{B}}^{* \perp} \rightarrow\left[\left(Z^{* \perp c}\right)^{2} / \varepsilon_{\infty}^{\perp c}\right]^{1 / 2}$ where $Z^{* \perp c}=2.71 e$ and $\varepsilon_{\infty}^{\perp c}=4.95$ are the Born effective charge and the experimental macroscopic dielectric constant, ${ }^{13}$ respectively. Similarly $e_{\mathrm{B}}^{* \|} \rightarrow$ $\left[\left(Z^{* \| c}\right)^{2} / \varepsilon_{\infty}^{\perp c}\right]^{1 / 2}$, with $Z^{* \| c}=0.82 e$ and $\varepsilon_{\infty}^{\| c}=4.10$. In order to restrict the contributions to long wavelengths, we will use a range parameter $\eta_{s}=2 / a^{2}$ in expression (9) (a similar role is played by the switching factor in real space in Ref. 24).

In the following it will be convenient to rewrite the dynamical matrix by separating off the nonregular Coulomb term:

$$
D(\vec{q})=R(\vec{q})+\left.\mathcal{C}(\vec{q}, \vec{\tau}=\overrightarrow{0})\right|_{1} .
$$

Here

$$
R(\vec{q})=F\left(\vec{q}_{\perp}\right)+J(\vec{q})+\bar{C}(\vec{q})
$$

is the "short-range" part. The regular Coulomb term $\bar{C}(\vec{q})$ is obtained from Eqs. (5)-(7) where only contributions with $\vec{\tau} \neq \overrightarrow{0}$ are retained. The nonregular term $\left.\mathrm{C}(\vec{q}, \vec{\tau}=\overrightarrow{0})\right|_{1}$ has the elements

$$
\left.\mathcal{C}_{i j}^{\kappa \kappa^{\prime}}(\vec{q}, \vec{\tau}=\overrightarrow{0})\right|_{1}=\frac{4 \pi e_{\kappa i}^{*} e_{\kappa^{\prime} j}^{*}}{v_{3 \mathrm{D}} \sqrt{m_{\kappa} m_{\kappa^{\prime}}}} \frac{q_{i} q_{j}}{|\vec{q}|^{2}} e^{-\frac{q^{2}}{4 \eta_{s}}}
$$

with $e_{\kappa i}^{*}=e_{\kappa}^{* \perp}$ or $e_{\kappa}^{* \|}$ for $i=1,2$ or $i=3$, respectively. We notice that a separation of terms as in Eq. (10) has to be made in first-principles calculations. ${ }^{39}$ The importance of the nonregular Coulomb term will become apparent in the next section where we study the phonon dispersion relations of $3 \mathrm{D}$ h-BN and analyze the LO-TO frequency splittings.

\section{PHONONS IN 3D HEXAGONAL BN}

The phonon dispersion relations are obtained from the solution of the secular equation

$$
\left|1 \omega^{2}-D(\vec{q})\right|=0 .
$$

Here 1 is the $12 \times 12$ unit matrix. The dynamical matrix is given by Eq. (10) where the covalent, van der Waals, and Coulomb terms are specified by Eqs. (3)-(12). Since only two-body forces are taken into account, the dynamical matrix is symmetric in the Cartesian indices, $D_{i j}^{\kappa \kappa^{\prime}}(\vec{q})=D_{j i}^{\kappa \kappa^{\prime}}(\vec{q})$. In the following we will give details on the used input parameters.

The matrix $F\left(\vec{q}_{\perp}\right)$ in Eq. (11) is based on a force constants model which was originally derived from inelastic $x$-ray scattering (IXS) work on graphite. ${ }^{40}$ The force constants mimic the in-plane covalent bonds. ${ }^{7}$ The force parameters $f_{r}^{(n)}, f_{i}^{(n)}$, and $f_{o}^{(n)}$ with $n=1,2, \ldots, 5$ refer to the radial (bond-stretching), in-plane, and out-of-plane tangential (bondbending) directions, respectively, of the $n$th neighbor atoms. This model was used to calculate the elastic properties 
TABLE I. Optical phonon frequencies in units of $\mathrm{cm}^{-1}$ at the $\Gamma$ point.

\begin{tabular}{lllllllll}
\hline \hline & $E_{2 g_{1}}$ & $B_{1 g_{1}}$ & $A_{2 u}(\mathrm{TO})$ & $A_{2 u}(\mathrm{LO})$ & $B_{1 g_{2}}$ & $E_{2 g_{2}}$ & $E_{1 u}(\mathrm{TO})$ & $E_{1 u}(\mathrm{LO})$ \\
\hline Present & 54.5 & 121.9 & 777.6 & 823.2 & 785.1 & 1309.6 & 1310.4 & 1542.3 \\
Ohba et al. $^{21}$ & 50 & 113 & 754 & 823 & 815 & 1382 & 1382 & 1614 \\
${\text { Serrano } \text { al } .^{29}}^{29}$ & 52 & 121 & 747 & & 809.7 & 1379 & 1378 & 1611 \\
Experiment & $52^{\mathrm{b}, \mathrm{c}}$ & & $783^{\mathrm{a}}$ & $828^{\mathrm{a}}$ & & $1366^{\mathrm{b}, \mathrm{c}}$ & $1367^{\mathrm{a}}$ & $1610^{\mathrm{a}}$ \\
& & & & & & $1370^{\mathrm{a}, \mathrm{d}}$ & & \\
\hline \hline
\end{tabular}

\footnotetext{
${ }^{\mathrm{a}}$ Geick et $a l .{ }^{13}$

${ }^{\mathrm{b}}$ Kuzuba et al. ${ }^{15}$

${ }^{\mathrm{c}}$ Nemanich et al. ${ }^{14}$

${ }^{\mathrm{d}}$ Reich et al. ${ }^{27}$
}

of graphene and graphite and the phonon dispersions of graphene multilayers. ${ }^{41}$ Recently, a similar model was applied to calculate the covalent part of the dynamical matrix of $2 \mathrm{D}$ h-BN, taking into account that the strength of the covalent bonds is weaker than in graphene. ${ }^{30}$ Here we will take the same numerical values of the force constants as in Table I of Ref. 30, except for the out-of-plane force constants $f_{o}^{(n)}$ which we multiply by a factor 0.9 .

The interplanar force constants $h_{i j}\left(\vec{n} \kappa ; \vec{n}^{\prime} \kappa^{\prime}\right)$ entering the van der Waals contribution $J(\vec{q})$ to the dynamical matrix, Eq. (4), are obtained from the second derivatives of an empirical Lennard-Jones potential,

$$
V(r)=\frac{\mathcal{B}}{r^{12}}-\frac{\mathcal{A}}{r^{6}},
$$

where $r=\left|\vec{X}\left(\vec{n}^{\prime} \kappa^{\prime}\right)-\vec{X}(\vec{n} \kappa)\right|$ is the interatomic distance. We take into account the interactions of a $B$ atom with the 2 nearest-neighbor $\mathrm{N}$ atoms at $(0,0, \pm c / 2)$, with 6 neighbor $\mathrm{B}$ atoms at $(a / \sqrt{3}, 0, \pm c / 2),(-a / 2 \sqrt{3}, \pm a / 2, \pm c / 2)$, and with 12 over-nearest $\mathrm{N}$ atoms at $( \pm a \sqrt{3} / 2, \pm a / 2, \pm c / 2)$, $(0, \pm a, \pm c / 2)$ in the two adjacent planes and vice versa. The constants $\mathcal{B}$ and $\mathcal{A}$ have the values $18.1 \times 10^{3} \mathrm{eV} \AA^{12}$ and $11.4 \mathrm{eV} \AA$, ${ }^{6}$ respectively, $1 / 4$ lower than those of graphite. ${ }^{42}$

The elements of the Coulomb matrix $C(\vec{q})$ have been given by Eqs. (5)-(12). We take ionic charges as specified in Sec. II.

The dynamical matrix $D(\vec{q})$ is Hermitian and hence the eigenvalues $\omega_{v}^{2}(\vec{q}), v=1, \ldots, 12$, are real. In addition the eigenvalues are positive and by taking the square root one

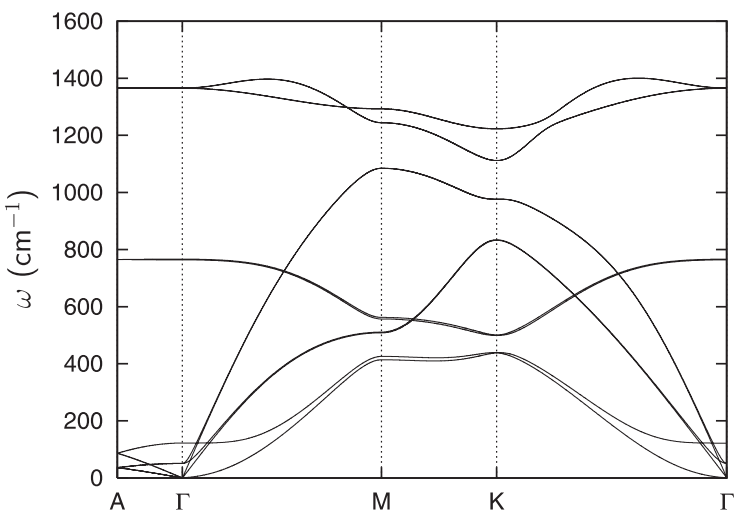

(a) obtains the phonon dispersion relations. In Fig. 3(a) we show the phonon dispersions without Coulomb forces; in Fig. 3(b) Coulomb forces are included. In calculating the dynamical matrix as a function of the wave vector, we have excluded the unphysical value at $\vec{q}=\overrightarrow{0}$ of the term $\left.\mathrm{C}_{i j}^{\kappa \kappa^{\prime}}(\vec{q}, \vec{\tau}=\overrightarrow{0})\right|_{1}$, Eq. (12).

For the space group $D_{6 h}^{4}$, the symmetry transformations of the zone-center optical phonons read ${ }^{13,14}$

$$
\Gamma=2 E_{2 g}+2 B_{1 g}+A_{2 u}+E_{1 u} .
$$

We recall that the $E_{2 g}$ modes are Raman active, the $A_{2 u}$ and $E_{1 u}$ modes are infrared active, and the $B_{1 g}$ modes are optically silent. ${ }^{13}$ Considering Fig. 3(b), we identify the zonecenter frequencies at $54.5 \mathrm{~cm}^{-1}(2 \times)$ and $121.9 \mathrm{~cm}^{-1}$ with $E_{2 g_{1}}$ (rigid-plane shears) and $B_{1 g_{1}}$ (rigid-plane compressions) modes, respectively. The frequency $\omega_{B_{1 g_{1}}}$ depends sensitively on the values of the van der Waals potential parameters $\mathcal{A}$ and $\mathcal{B}$. The modes $A_{2 u}$ and $B_{1 g_{2}}$ involve intraplane shear displacements parallel to the $\vec{c}$ axis. We obtain $\omega_{B_{1 g_{2}}}=$ $785.1 \mathrm{~cm}^{-1}$ at $\Gamma$ on both sides $\overline{\Gamma A}$ and $\overline{\Gamma M}$. However the mode $A_{2 u}$ has a discontinuity at $\Gamma$ with values $\omega_{A_{2 u}}=777.6 \mathrm{~cm}^{-1}$ and $823.2 \mathrm{~cm}^{-1}$ on the sides $\overline{\Gamma M}$ and $\overline{\Gamma A}$, respectively. This discontinuity is due to the nonregular Coulomb term, Eq. (12), which vanishes along $\overline{\Gamma M}$ (transverse $A_{2 u}$ mode) but differs from zero along $\overline{\Gamma A}$ (longitudinal $A_{2 u}$ mode). Of interest are also the cases of the in-plane shear modes. We find a degenerate $E_{2 g_{2}}$ mode with $\omega_{E_{2 g_{2}}}=1309.6 \mathrm{~cm}^{-1}$ on both sides of $\Gamma$, and an $E_{1 u}$ mode which is degenerate with frequency $\omega_{E_{1 u}}(\mathrm{TO})=$

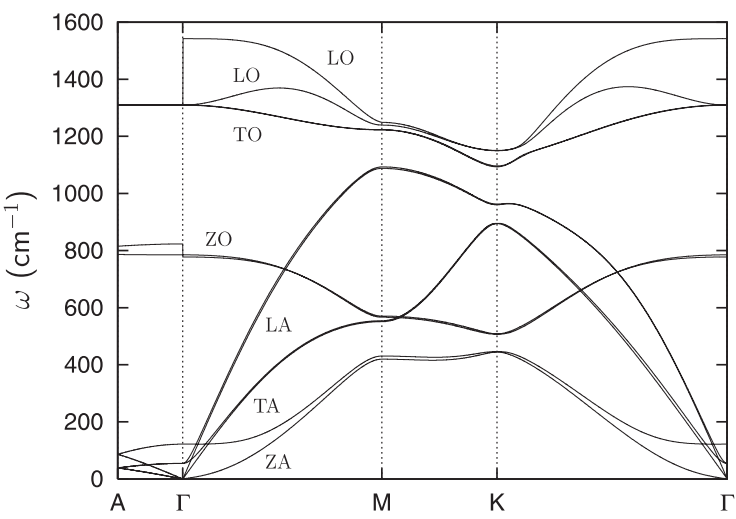

(b)

FIG. 3. Phonon dispersions of 3D h-BN (a) without Coulomb forces and (b) with Coulomb forces included. 
$1310.4 \mathrm{~cm}^{-1}$ on the side $\overline{\Gamma A}$ and split with $\omega_{E_{1 u}}(\mathrm{TO})=$ $1310.4 \mathrm{~cm}^{-1}$ and $\omega_{E_{1 u}}(\mathrm{LO})=1542.3 \mathrm{~cm}^{-1}$ on the sides $\overline{\Gamma M}$ and $\overline{\Gamma K}$ at $\Gamma$. Our results are in full qualitative agreement with the TO and LO phonon frequencies calculated at the $\Gamma$ point by first-principles calculations. ${ }^{21}$ The quantitative differences are less than $10 \%$. The calculated splitting $\Delta \omega_{E_{1 u}}=\omega_{E_{1 u}}(\mathrm{LO})-$ $\omega_{E_{1 u}}$ (TO) of the $E_{1 u}$ mode at $\Gamma$ amounts to $231.9 \mathrm{~cm}^{-1}$, close to the values measured by infrared experiments ${ }^{13}$ and calculated by ab initio theory. ${ }^{20,21,25,29}$ Also the discontinuity $\Delta \omega_{A_{2 u}}=\omega_{A_{2 u}}(\mathrm{LO})-\omega_{A_{2 u}}(\mathrm{TO})=45.6 \mathrm{~cm}^{-1}$ of the $A_{2 u}$ mode at $\Gamma$ is close to the experimental value. ${ }^{13}$ In Table I we give a comparison of our calculated phonon frequencies at the $\Gamma$ point with experimental data and ab initio calculation results.

We now show that the splitting of the $E_{1 u}$ mode is due to the long-range Coulomb term, Eq. (12), which acts as a symmetrybreaking field. This feature is similar to the well-known TO-LO splitting in cubic ionic crystals. ${ }^{36}$ Going back to Eq. (10) we will treat $\left.\mathcal{C}\right|_{1}$ as a perturbation, assuming that the doubly degenerate eigenvalue of $R(\vec{q}=\overrightarrow{0})$ belonging to the $E_{1 u}$ mode, $\omega_{E_{1 u}}^{2}(\mathrm{TO})$, is known. The corresponding eigenvectors $\vec{\xi}^{(v)}(\kappa i)$, with $v \in\{1,2\}, \kappa \in\left\{\mathrm{B}, \mathrm{N}, \mathrm{B}^{\prime}, \mathrm{N}^{\prime}\right\}$, and $i \in\{x, y, z\}$, read

$$
\begin{aligned}
\vec{\xi}^{(1)}(\kappa i)= & \sqrt{\frac{\mu}{2}}\left(\frac{1}{\sqrt{m_{\mathrm{B}}}}, 0,0,-\frac{1}{\sqrt{m_{\mathrm{N}}}}, 0,0, \frac{1}{\sqrt{m_{\mathrm{B}}}}, 0,0\right. \\
& \left.-\frac{1}{\sqrt{m_{\mathrm{N}}}}, 0,0\right), \\
\vec{\xi}^{(2)}(\kappa i)= & \sqrt{\frac{\mu}{2}}\left(0, \frac{1}{\sqrt{m_{\mathrm{B}}}}, 0,0,-\frac{1}{\sqrt{m_{\mathrm{N}}}}, 0,0, \frac{1}{\sqrt{m_{\mathrm{B}}}}, 0,0\right. \\
& \left.-\frac{1}{\sqrt{m_{\mathrm{N}}}}, 0\right) .
\end{aligned}
$$

Here $\vec{\xi}^{(1)}$ and $\vec{\xi}^{(2)}$ are proportional to the $x$ and the $y$ components of the intraplane optical displacements; $\mu=$ $m_{\mathrm{B}} m_{\mathrm{N}} /\left(m_{\mathrm{B}}+m_{\mathrm{N}}\right)$ is the in-plane reduced mass. These displacements generate electric dipoles. The resulting polarization gives rise to a longitudinal macroscopic electric field.

We project the matrix $R$ onto the subspace of $E_{1 u}$ symmetry and write

$$
\begin{aligned}
\hat{R}_{\nu v^{\prime}}(\vec{q}=\overrightarrow{0}) & =\sum_{\kappa \kappa^{\prime}} \sum_{i j} \xi^{(v)}(\kappa i) R_{i j}^{\kappa \kappa^{\prime}}(\vec{q}=\overrightarrow{0}) \xi^{\left(\nu^{\prime}\right)}\left(\kappa^{\prime} j\right) \\
& =\delta_{v v^{\prime}} \omega_{E_{1 u}}(\mathrm{TO})^{2}
\end{aligned}
$$

where the circumflex indicates the new basis.

Similarly the matrix elements of the nonregular Coulomb term in the $E_{1 u}$ basis are given by

$$
\left.\hat{\mathrm{C}}_{\nu \nu^{\prime}}(\vec{q})\right|_{1}=\left.\sum_{\kappa \kappa^{\prime}} \sum_{i j} \xi^{(\nu)}(\kappa i) \mathcal{C}_{i j}^{\kappa \kappa^{\prime}}(\vec{q}, \vec{\tau}=\overrightarrow{0})\right|_{1} \xi^{\left(\nu^{\prime}\right)}\left(\kappa^{\prime} j\right) .
$$

Explicitly we find

$$
\left.\hat{\mathrm{C}}_{v v^{\prime}}(\vec{q})\right|_{1}=\frac{8 \pi\left(e_{\mathrm{B}}^{* \perp}\right)^{2}}{v_{3 \mathrm{D}}} \frac{q_{v} q_{v^{\prime}}}{|\vec{q}|^{2}},
$$

where $q_{v}\left(q_{v^{\prime}}\right)$ stands for $q_{x}$ if $v\left(v^{\prime}\right)=1$ or for $q_{y}$ if $v\left(v^{\prime}\right)=2$. Applying perturbation theory for the degenerate unperturbed eigenvalues, we solve the $2 \times 2$ secular determinant:

$$
\left|\hat{\mathrm{C}}_{\nu v^{\prime}}(\vec{q})\right|_{1}+\delta_{v v^{\prime}}\left(\omega_{E_{1 u}}(\mathrm{TO})^{2}-\omega^{2}\right) \mid=0
$$

For $\vec{q} \rightarrow \overrightarrow{0}$ along $\overline{\Gamma K}$ or $\overline{\Gamma M}$ in the basal plane of the BZ the square roots of the two eigenvalues read

$$
\begin{gathered}
\omega^{(1)}=\omega_{E_{1 u}}(\mathrm{TO}), \\
\omega^{(2)}=\omega_{E_{1 u}}(\mathrm{LO})=\sqrt{\omega_{E_{1 u}}(\mathrm{TO})^{2}+\frac{8 \pi\left(e_{\mathrm{B}}^{* \perp}\right)^{2}}{v_{3 \mathrm{D}} \mu}} .
\end{gathered}
$$

Inserting the numerical values for the quantities $e_{\mathrm{B}}^{* \perp}, \omega_{E_{1 u}}(\mathrm{TO})$, $v_{3 \mathrm{D}}$, and $\mu$, we obtain $\omega^{(2)}=1542.1 \mathrm{~cm}^{-1}$. The LO-TO frequency splitting $\Delta \omega=\omega^{(2)}-\omega^{(1)}$ is found to be $231.9 \mathrm{~cm}^{-1}$, which exactly matches the value obtained from the numerical solution of the $12 \times 12$ secular equation. One easily shows that the eigenvectors corresponding to the degenerate mode of $E_{2 g_{2}}$ symmetry at $\Gamma$ give no splitting. Those eigenvectors are obtained from $\vec{\xi}^{(1)}$ and $\vec{\xi}^{(2)}$ by changing the sign of the 7 th and 10th components and 8th and 11th components, respectively. The electric dipoles in adjacent planes are of opposite sign and the resulting polarization vanishes.

We finally investigate the discontinuity of the $A_{2 u}$ mode (out-of-plane displacements) which appears as a LO and a TO branch on the sides $\overline{\Gamma A}$ and $\overline{\Gamma M}(\overline{\Gamma K})$, respectively, in Fig. 3(b). We introduce the eigenvector

$$
\begin{aligned}
\vec{\xi}^{(3)}(\kappa i)= & \sqrt{\frac{\mu}{2}}\left(0,0, \frac{1}{\sqrt{m_{\mathrm{B}}}}, 0,0,-\frac{1}{\sqrt{m_{\mathrm{N}}}}, 0,0, \frac{1}{\sqrt{m_{\mathrm{B}}}}, 0,0\right. \\
& \left.-\frac{1}{\sqrt{m_{\mathrm{N}}}}\right)
\end{aligned}
$$

and calculate the matrix elements $\hat{R}_{33}$ and $\hat{\mathrm{C}}_{33}$. Solution of the perturbation problem gives for $\vec{q} \rightarrow \overrightarrow{0}$ along $\overline{\Gamma M}(\overline{\Gamma K})$

$$
\omega_{1}^{(3)} \equiv \omega_{A_{2 u}}(\mathrm{TO})
$$

and for $\vec{q} \rightarrow \overrightarrow{0}$ along $\overline{\Gamma A}$

$$
\omega_{2}^{(3)} \equiv \omega_{A_{2 u}}(\mathrm{LO})=\sqrt{\omega_{A_{2 u}}(\mathrm{TO})^{2}+\frac{8 \pi\left(e_{\mathrm{B}}^{* \|}\right)^{2}}{v_{3 \mathrm{D}} \mu}} .
$$

Numerical evaluation then leads to $\omega_{2}^{(3)}=823.2 \mathrm{~cm}^{-1}$ whence the discontinuity amounts to $\Delta \omega_{A_{2 u}}=\omega_{2}^{(3)}-\omega_{1}^{(3)}=$ $45.6 \mathrm{~cm}^{-1}$, again in agreement with the numerical solution of the secular problem.

Comparing the present results [Fig. 3(b)] with the experimental IXS phonon dispersion relations, see Fig. 2(a) of Ref. 29, and the corresponding ab initio calculations, ${ }^{20,29,43}$ we see an overall satisfactory agreement for the phonon branches ZA (acoustic out-of-plane displacements), TA (transverse acoustic), LA (longitudinal acoustic), and ZO (optical out-ofplane). As a quantitive difference we realize that the $\mathrm{ZA}$ branch in our calculations along the path $\overline{M K}$ in the $\mathrm{BZ}$ is higher by an amount $\approx 100 \mathrm{~cm}^{-1}$ than in the ab initio calculations. However, our results with an average ZA $(\mathrm{M}-\mathrm{K})$ frequency $\approx 440 \mathrm{~cm}^{-1}$ are closer to $\approx 460 \mathrm{~cm}^{-1}$ observed experimentally by resonant Raman scattering. ${ }^{27}$

Considering the high-frequency optical branches which correspond to in-plane displacements we notice in Fig. 3(b) that in addition to the splitting of the $E_{1 u}$ mode at $\Gamma$, the $E_{2 g_{2}}$ mode, which is degenerate at $\Gamma$, evolves into two branches away from $\Gamma$ to $M(K)$; the lower branch coincides almost with the lowest $E_{1 u}$ branch while the higher $E_{2 g_{2}}$ branch 
crosses the lower $E_{1 u}$ branch and shows a pronounced overbending. The amount of overbending of $\approx 60 \mathrm{~cm}^{-1}$ is smaller than $\approx 100 \mathrm{~cm}^{-1}$ predicted by ab initio calculated dispersion relations $^{20}$ and measured by resonant Raman scattering. ${ }^{27}$ This quantitative difference is probably due to our simplified treatment of the ionic charge deformations.

\section{MULTILAYERS}

Here we study the lattice dynamics of a h-BN slab which consists of a finite number $\mathcal{N}$ of $2 \mathrm{D}$ h-BN layers. The layers (atomic planes) are superposed in the same pattern (002) as in the 3D crystal. Since the slab is infinitely extended only in two dimensions perpendicular to the $\vec{c}$ axis, the formalism of 3D Fourier transforms is no longer valid. We have to use 2D Fourier transforms with wave vectors $\vec{q}_{\perp}=\left(q_{x}, q_{y}\right)$ similar to the case of the monolayer problem; ${ }^{30}$ in addition we have to describe the superposition of layers in direct space. Equivalently we consider the slab as a "2D" crystal which consists of prismatic unit cells with basis vectors $\vec{a}_{1}, \vec{a}_{2}$ perpendicular to $\vec{c}$. Each unit cell contains $\mathcal{N}$ pairs of atoms $\mathrm{B}, \mathrm{N}\left(\mathrm{B}^{\prime}, \mathrm{N}^{\prime}\right)$. We ascribe to each pair of atoms an effective

$$
\Delta_{\mathcal{N}}\left(\vec{q}_{\perp}\right)=\left(\begin{array}{cc}
\mathcal{D}\left(0,0 \mid \vec{q}_{\perp}\right) & \mathcal{D}\left(0,1 \mid \vec{q}_{\perp}\right) \\
\mathcal{D}\left(1,0 \mid \vec{q}_{\perp}\right) & \mathcal{D}\left(1,1 \mid \vec{q}_{\perp}\right) \\
\mathcal{D}\left(2,0 \mid \vec{q}_{\perp}\right) & \mathcal{D}\left(2,1 \mid \vec{q}_{\perp}\right) \\
\vdots & \vdots \\
\mathcal{D}\left(\mathcal{N}-1,0 \mid \vec{q}_{\perp}\right) & \mathcal{D}\left(\mathcal{N}-1,1 \mid \vec{q}_{\perp}\right)
\end{array}\right.
$$

Here the "same-plane" matrices $\mathcal{D}\left(l, l \mid \vec{q}_{\perp}\right)$ are given by

$$
\mathcal{D}\left(l, l \mid \vec{q}_{\perp}\right)=D\left(l, l \mid \vec{q}_{\perp}\right)+K\left(l, l \mid \vec{q}_{\perp}=\overrightarrow{0}\right)
$$

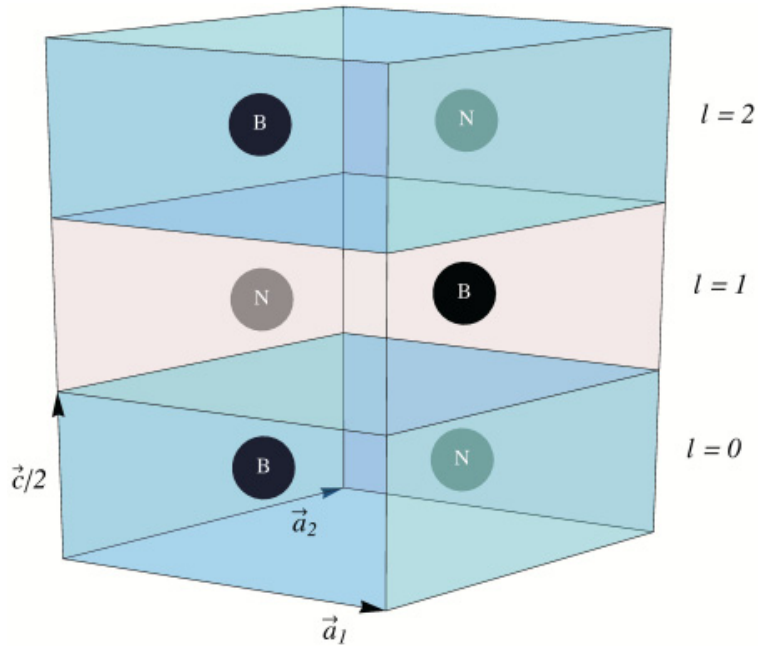
slab.

FIG. 4. (Color online) Prismatic unit cell for a three-layer h-BN volume $v_{1}=\left(\vec{a}_{1} \times \vec{a}_{2}\right) \cdot \vec{c} / 2=a^{2} c \sqrt{3} / 4$. See Fig. 4 for the case $\mathcal{N}=3$ with unit cell volume $v_{3}=3 v_{1}$. Labeling the layers by an index $l \in\{0,1,2, \ldots, \mathcal{N}-1\}$, we write for the positions of the atoms

$$
\vec{X}^{\kappa}\left(\vec{n}_{\perp}, l\right)=\vec{X}\left(\vec{n}_{\perp}\right)+\vec{r}^{\kappa}(l) .
$$

Here $\vec{X}\left(\vec{n}_{\perp}\right)=n_{1} \vec{a}^{(1)}+n_{2} \vec{a}^{(2)}$ are the position vectors of the unit cells; $\vec{n}_{\perp}=\left(n_{1}, n_{2}\right)$ and $\vec{a}^{(1)}=(\sqrt{3} a / 2, a / 2,0), \vec{a}^{(2)}=$ $(\sqrt{3} a / 2,-a / 2,0)$. The positions of the atoms in the unit cell depend on the layer index $l$. One has $\vec{r}^{\kappa}(l)=(0,0, l c / 2)$ and $\vec{r}^{\kappa}(l)=(a / \sqrt{3}, 0, l c / 2)$ where for $l$ even $\kappa=B$ and $\mathrm{N}$ respectively while for $l$ uneven $\kappa=\mathrm{N}$ and $\mathrm{B}$ respectively (compare $\mathrm{N}^{\prime}$ and $\mathrm{B}^{\prime}$ in Sec. II).

We recall that the dynamical matrix of the monolayer case $2 \mathrm{D} \mathrm{h}-\mathrm{BN}$ is of dimension $6 \times 6$. The dynamical matrix of the $\mathcal{N}$ layer problem $\Delta_{\mathcal{N}}\left(\vec{q}_{\perp}\right)$ is of dimension $6 \mathcal{N} \times 6 \mathcal{N}$. It is convenient to formulate the dynamical matrix in terms of $\mathcal{N}^{2}$ submatrices $\mathcal{D}\left(l, l^{\prime} \mid \vec{q}_{\perp}\right)$ of dimension $6 \times 6$, where $l\left(l^{\prime}\right)$ $\in\{0,1, \ldots, \mathcal{N}-1\}$. Thereby we take into account interactions within a same layer $\left(l=l^{\prime}\right)$ and interactions between layers separated by a distance $\left|l^{\prime}-l\right| c / 2, l \neq l^{\prime}$ :

$$
\left.\begin{array}{ccc}
\mathcal{D}\left(0,2 \mid \vec{q}_{\perp}\right) & \ldots & \mathcal{D}\left(0, \mathcal{N}-1 \mid \vec{q}_{\perp}\right) \\
\mathcal{D}\left(1,2 \mid \vec{q}_{\perp}\right) & \ldots & \mathcal{D}\left(1, \mathcal{N}-1 \mid \vec{q}_{\perp}\right) \\
\mathcal{D}\left(2,2 \mid \vec{q}_{\perp}\right) & \ldots & \mathcal{D}\left(2, \mathcal{N}-1 \mid \vec{q}_{\perp}\right) \\
\vdots & \ddots & \vdots \\
\mathcal{D}\left(\mathcal{N}-1,2 \mid \vec{q}_{\perp}\right) & \ldots & \mathcal{D}\left(\mathcal{N}-1, \mathcal{N}-1 \mid \vec{q}_{\perp}\right)
\end{array}\right)
$$

where the first term on the rhs is the dynamical matrix of the $l$ th monolayer. Assuming that the in-plane interactions are the same for all planes, one has in terms of elements

$$
D_{i j}^{\kappa \kappa^{\prime}}\left(l, l \mid \vec{q}_{\perp}\right)=F_{i j}^{\kappa \kappa^{\prime}}\left(l, l \mid \vec{q}_{\perp}\right)+C_{i j}^{\kappa \kappa^{\prime}}\left(l, l \mid \vec{q}_{\perp}\right),
$$

where $F$ and $C$ stand for the intraplane covalent and Coulomb interactions, respectively. ${ }^{30}$ From the correlation between $l$ and $\kappa$ [see Eq. (25)], it follows that $D\left(l, l \mid \vec{q}_{\perp}\right)=D(l-$ $\left.1, l-1 \mid \vec{q}_{\perp}\right)^{*}$. The term $K\left(l, l \mid \vec{q}_{\perp}=\overrightarrow{0}\right)$ on the rhs of Eq. (27) accounts for the self-interaction (crystal field) due to interplane couplings [see Eq. (39) below].

The interplane coupling matrices $\mathcal{D}\left(l, l^{\prime} \mid \vec{q}_{\perp}\right)$ with $l \neq l^{\prime}$ in Eq. (26) are due to van der Waals and Coulomb contributions. One has

$$
\mathcal{D}_{i j}^{\kappa \kappa^{\prime}}\left(l, l^{\prime} \mid \vec{q}_{\perp}\right)=J_{i j}^{\kappa \kappa^{\prime}}\left(\vec{q}_{\perp}\right) \delta_{l, l^{\prime} \pm 1}+C_{i j}^{\kappa \kappa^{\prime}}\left(l, l^{\prime} \mid \vec{q}_{\perp}\right) .
$$

The van der Waals forces are effective between nearest neighbor planes similar to the case of graphene multilayers. ${ }^{43}$ The elements $J_{i j}^{\kappa \kappa^{\prime}}\left(\vec{q}_{\perp}\right)$ are obtained from Eq. (4) with $\vec{q}$ and $\vec{n}^{\prime}$ replaced by the planar vectors $\vec{q}_{\perp}$ and $\vec{n}_{\perp}^{\prime}$, respectively. The Coulomb matrix $C\left(l, l^{\prime} \mid \vec{q}_{\perp}\right)$ accounts for all interactions between ions belonging to different planes $l$ and $l^{\prime}$. We write

$$
\begin{aligned}
C_{i j}^{\kappa \kappa^{\prime}}\left(l, l^{\prime} \mid \vec{q}_{\perp}\right)= & \frac{1}{\sqrt{m_{\kappa}(l) m_{\kappa^{\prime}}\left(l^{\prime}\right)}}\left[\left.\Phi_{i j}^{\kappa \kappa^{\prime}}\left(l, l^{\prime} \mid \vec{q}_{\perp}\right)\right|_{1}\right. \\
& \left.+\left.\Phi_{i j}^{\kappa \kappa^{\prime}}\left(l, l^{\prime} \mid \vec{q}_{\perp}\right)\right|_{2}\right],
\end{aligned}
$$


where the coupling parameters $\left.\Phi\right|_{1}$ and $\left.\Phi\right|_{2}$ are calculated by means of the Ewald method. For details of the derivation we refer to the Appendix. Here we quote the results. For in-plane displacements, $i, j \in\{x, y\}$, we obtain by means of Eqs. (A9) and (A10)

$$
\begin{aligned}
& \left.\Phi_{i j}^{\kappa \kappa^{\prime}}\left(l, l^{\prime} \mid \vec{q}_{\perp}\right)\right|_{1}=\frac{\pi}{v_{2 \mathrm{D}}} e_{\kappa}^{\perp} e_{\kappa^{\prime}}^{\perp} \sum_{\vec{\tau}_{\perp}} \frac{\left(\vec{\tau}_{\perp}-\vec{q}_{\perp}\right)_{i}\left(\vec{\tau}_{\perp}-\vec{q}_{\perp}\right)_{j}}{\left|\vec{\tau}_{\perp}-\vec{q}_{\perp}\right|} \\
& \times e^{\left.i \vec{\tau}_{\perp} \cdot \vec{r}^{\kappa^{\prime}}\left(l^{\prime}\right)-\vec{r}^{k}(l)\right]} \mathcal{L}\left(\left|l^{\prime}-l\right| ;\left|\vec{\tau}_{\perp}-\vec{q}_{\perp}\right|\right),
\end{aligned}
$$

where, with $\left|l^{\prime}-l\right|=|\lambda|,|\lambda|=0,1,2, \ldots, \mathcal{N}-1$,

$$
\begin{aligned}
\mathcal{L}\left(|\lambda| ;\left|\vec{\tau}_{\perp}-\vec{q}_{\perp}\right|\right)= & e^{\lambda \frac{c}{2}\left|\vec{\tau}_{\perp}-\vec{q}_{\perp}\right|} \operatorname{erfc}\left[\frac{\left|\vec{\tau}_{\perp}-\vec{q}_{\perp}\right|}{2 \sqrt{\eta}}+\lambda \frac{c}{2} \sqrt{\eta}\right] \\
& +e^{-\lambda \frac{c}{2}\left|\vec{\tau}_{\perp}-\vec{q}_{\perp}\right|} \operatorname{erfc}\left[\frac{\left|\vec{\tau}_{\perp}-\vec{q}_{\perp}\right|}{2 \sqrt{\eta}}-\lambda \frac{c}{2} \sqrt{\eta}\right] .
\end{aligned}
$$

Here $\vec{\tau}_{\perp}$ is a $2 \mathrm{D}$ reciprocal lattice vector; $v_{2 \mathrm{D}}=a^{2} \sqrt{3} / 2$ is the area of the 2D unit cell.

The second term on the rhs of Eq. (30) reads for $i, j \in\{x, y\}$

$$
\begin{aligned}
\left.\Phi_{i j}^{\kappa \kappa^{\prime}}\left(l, l^{\prime} \mid \vec{q}_{\perp}\right)\right|_{2}= & -e_{\kappa}^{\perp} e_{\kappa^{\prime}}^{\perp} \eta^{\frac{3}{2}} \sum_{\vec{n}_{\perp}^{\prime}} H_{i j}^{\kappa \kappa^{\prime}}\left(\sqrt{\eta} \mid \vec{X}^{\kappa^{\prime}}\left(\vec{n}_{\perp}^{\prime}, l^{\prime}\right)\right. \\
& \left.-\vec{X}^{\kappa}\left(\vec{n}_{\perp}, l\right) \mid\right) e^{i \vec{q}_{\perp} \cdot\left[\vec{X}^{\kappa^{\prime}}\left(\vec{n}_{\perp}^{\prime}, l^{\prime}\right)-\vec{X}^{\kappa}\left(\vec{n}_{\perp}, l\right)\right]},
\end{aligned}
$$

where $H_{i j}^{\kappa \kappa^{\prime}}$ is still defined by Eq. (8b) and where the summation runs over all lattice points $\vec{n}_{\perp}^{\prime}$ of the plane $l^{\prime}$.

The coupling parameters due to out-of-plane displacements are given by [see Eq. (A11)]

$$
\begin{aligned}
\left.\Phi_{z z}^{\kappa \kappa^{\prime}}\left(l, l^{\prime} \mid \vec{q}_{\perp}\right)\right|_{1}= & \frac{2 \pi}{v_{2 \mathrm{D}}} e_{\kappa}^{\|} e_{\kappa^{\prime}}^{\|} \sum_{\vec{\tau}_{\perp}}\left[2 \sqrt{\frac{\eta}{\pi}} e^{-\left[\left|\vec{\tau}_{\perp}-\vec{q}_{\perp}\right|^{2} / 4 \eta+\left(l^{\prime}-l\right) c^{2} \eta / 4\right]}\right. \\
& \left.-\frac{\left|\vec{\tau}_{\perp}-\vec{q}_{\perp}\right|}{2} \mathcal{L}\left(\left|l^{\prime}-l\right| ;\left|\vec{\tau}_{\perp}-\vec{q}_{\perp}\right|\right)\right] \\
& \times e^{i \vec{\tau}_{\perp} \cdot\left[\vec{r}^{\kappa^{\prime}}\left(l^{\prime}\right)-\vec{r}^{\kappa}(l)\right]}
\end{aligned}
$$

and

$$
\begin{aligned}
\left.\Phi_{z z}^{\kappa \kappa^{\prime}}\left(l, l^{\prime} \mid \vec{q}_{\perp}\right)\right|_{2}= & -e_{\kappa}^{\|} e_{\kappa^{\prime}}^{\|} \eta^{\frac{3}{2}} \sum_{\vec{n}_{\perp}^{\prime}} H_{z z}^{\kappa \kappa^{\prime}}\left(\sqrt{\eta} \mid \vec{X}^{\kappa^{\prime}}\left(\vec{n}_{\perp}^{\prime}, l^{\prime}\right)\right. \\
& \left.-\vec{X}^{\kappa}\left(\overrightarrow{0}_{\perp}, l\right) \mid\right) e^{i \vec{q}_{\perp} \cdot\left[\vec{X}^{\kappa^{\prime}}\left(\vec{n}_{\perp}^{\prime}, l^{\prime}\right)-\vec{X}^{\kappa}\left(\overrightarrow{0}_{\perp}, l\right)\right]} .
\end{aligned}
$$

Here the function $H_{z z}^{\kappa \kappa^{\prime}}$ is defined by Eq. (8b).

Finally we consider the mixed terms with in- and out-ofplane displacements $i \in\{x, y\}$ and $j=z$, respectively. From Eq. (A12) we obtain

$$
\begin{aligned}
\left.\Phi_{i z}^{\kappa \kappa^{\prime}}\left(l, l^{\prime} \mid \vec{q}_{\perp}\right)\right|_{1}= & \frac{-i 2 \pi}{v_{2 \mathrm{D}}} e_{\kappa}^{\perp} e_{\kappa^{\prime}}^{\|} \sum_{\vec{\tau}_{\perp}}\left(\vec{\tau}_{\perp}-\vec{q}_{\perp}\right)_{i} e^{i \tau_{\perp} \cdot\left[\vec{r}^{k^{\prime}}\left(l^{\prime}\right)-\vec{r}^{\kappa}(l)\right]} \\
& \times \mathcal{P}\left(\left|l^{\prime}-l\right| ;\left|\vec{\tau}_{\perp}-\vec{q}_{\perp}\right|\right)
\end{aligned}
$$

with

$$
\begin{aligned}
\mathcal{P}\left(|\lambda| ;\left|\vec{\tau}_{\perp}-\vec{q}_{\perp}\right|\right) & \\
= & e^{\lambda \frac{c}{2}\left|\vec{\tau}_{\perp}-\vec{q}_{\perp}\right|}\left\{\frac{1}{2} \operatorname{erfc}\left(\frac{\left|\vec{\tau}_{\perp}-\vec{q}_{\perp}\right|}{2 \sqrt{\eta}}+\lambda \frac{c}{2} \sqrt{\eta}\right)-\frac{1}{\left|\vec{\tau}_{\perp}-\vec{q}_{\perp}\right|} \sqrt{\frac{\eta}{\pi}} e^{-\left[\frac{\left|\vec{\tau}_{\perp}-\vec{q}_{\perp}\right|}{2 \sqrt{\eta}}+\lambda \frac{c}{2} \sqrt{\eta}\right]}\right\} \\
& -e^{-\lambda \frac{c}{2}\left|\vec{\tau}_{\perp}-\vec{q}_{\perp}\right|}\left\{\frac{1}{2} \operatorname{erfc}\left(\frac{\left|\vec{\tau}_{\perp}-\vec{q}_{\perp}\right|}{2 \sqrt{\eta}}-\lambda \frac{c}{2} \sqrt{\eta}\right)-\frac{1}{\left|\vec{\tau}_{\perp}-\vec{q}_{\perp}\right|} \sqrt{\frac{\eta}{\pi}} e^{-\left[\frac{\left|\vec{\tau}_{\perp}-\vec{q}_{\perp}\right|}{2 \sqrt{\eta}}-\lambda \frac{c}{2} \sqrt{\eta}\right]}\right\} .
\end{aligned}
$$

The term $\left.\Phi_{i z}^{\kappa \kappa^{\prime}}\left(l, l^{\prime} \mid \vec{q}_{\perp}\right)\right|_{2}$ is obtained as

$$
\begin{aligned}
\left.\Phi_{i z}^{\kappa \kappa^{\prime}}\left(l, l^{\prime} \mid \vec{q}_{\perp}\right)\right|_{2} \\
=-e_{\kappa}^{\perp} e_{\kappa^{\prime}}^{\|} \eta^{\frac{3}{2}} \sum_{\vec{n}_{\perp}^{\prime}} H_{i z}^{\kappa \kappa^{\prime}} \times\left(\sqrt{\eta}\left|\vec{X}^{\kappa^{\prime}}\left(\vec{n}_{\perp}^{\prime}, l^{\prime}\right)-\vec{X}^{\kappa}\left(\overrightarrow{0}_{\perp}, l\right)\right|\right) \\
\quad \times e^{i \vec{q}_{\perp} \cdot\left[\vec{X}^{\kappa^{\prime}}\left(\vec{n}_{\perp}^{\prime}, l^{\prime}\right)-\vec{X}^{\kappa}\left(\overrightarrow{0}_{\perp}, l\right)\right]} .
\end{aligned}
$$

For the case $l=l^{\prime}$, the coupling parameters $\left.\Phi_{i j}^{\kappa \kappa^{\prime}}\left(l, l^{\prime} \mid \vec{q}_{\perp}\right)\right|_{1,2}$, $i(j) \in\{x, y, z\}$, reduce to the corresponding expressions for the monolayer ${ }^{30}$ and in addition the functions $\Phi_{i z}^{\kappa \kappa^{\prime}}$ vanish.

Having obtained the interplane coupling parameters $\mathcal{D}\left(l, l^{\prime} \mid \vec{q}_{\perp}\right)$ we determine the self-interaction term $K\left(l, l \mid \vec{q}_{\perp}=\right.$ $\overrightarrow{0})$, Eq. (27). Only elements with $\kappa=\kappa^{\prime}$ are nonvanishing:

$$
K_{i j}^{\kappa \kappa}\left(l, l \mid \vec{q}_{\perp}=\overrightarrow{0}\right)=-\sum_{l^{\prime}=0}^{\mathcal{N}-1}, \sum_{\kappa^{\prime}} \mathcal{D}_{i j}^{\kappa \kappa^{\prime}}\left(l, l^{\prime} \mid \vec{q}_{\perp}=\overrightarrow{0}\right) .
$$

The prime on the first summation on the rhs indicates that $l^{\prime}=l$ is excluded. The self-interactions due to the in-plane forces have been included already in the monolayer terms $D\left(l, l \mid \vec{q}_{\perp}\right)$.

The dynamical matrix $\Delta_{\mathcal{N}}\left(\vec{q}_{\perp}\right)$ is Hermitian and, due to the two-body forces nature of the interaction, its elements are again symmetric in the Cartesian displacement indices $i, j$. The hermiticity then reads

$$
\mathcal{D}_{i j}^{\kappa \kappa^{\prime}}\left(l, l^{\prime} \mid \vec{q}_{\perp}\right)=\mathcal{D}_{i j}^{\kappa^{\prime} \kappa}\left(l^{\prime}, l \mid \vec{q}_{\perp}\right)^{*}
$$

(for both cases $l=l^{\prime}$ and $l \neq l^{\prime}$ ).

Before calculating the phonon dispersion relations some comments on the long-wavelength behavior of the Coulomb term are in order. We recall that in $3 \mathrm{D} \mathrm{h}-\mathrm{BN}$ the nonregular Coulomb term $\left.\mathcal{C}_{i j}^{\kappa \kappa^{\prime}}(\vec{q}, \vec{\tau}=\overrightarrow{0})\right|_{1}$, Eq. (12), leads to the LO-TO splitting of the $E_{1 u}$ mode and to the discontinuity of the $A_{2 u}$ mode at $\Gamma$. In the multilayer case we consider the long-wavelength contribution to the dynamical matrix arising from the term with $\vec{\tau}_{\perp}=\overrightarrow{0}$ in Eq. (31). By means of Eq. (31) 
we obtain

$$
\begin{aligned}
\left.\mathcal{C}_{i j}^{\kappa \kappa^{\prime}}\left(l, l^{\prime} \mid \vec{q}_{\perp}, \vec{\tau}=\overrightarrow{0}\right)\right|_{1}= & \frac{\pi e_{\kappa}^{* \perp} e_{\kappa^{\prime}}^{* \perp}}{v_{2 \mathrm{D}} \sqrt{m_{\kappa}(l) m_{\kappa^{\prime}}\left(l^{\prime}\right)}} \frac{q_{i} q_{j}}{\left|\vec{q}_{\perp}\right|} \\
& \times \mathcal{L}\left(\left|l^{\prime}-l\right| ;\left|\vec{q}_{\perp}\right|\right) .
\end{aligned}
$$

Here we have accounted for the deformation of the ions at long wavelength by taking the effective charges $e_{\kappa}^{* \perp}$ (see Sec. II). $\left|\vec{q}_{\perp}\right|$,

In the long-wavelength regime one has, up to first order in

$$
\mathcal{L}\left(|\lambda| ;\left|\vec{q}_{\perp}\right|\right)=2\left[1-\left|\vec{q}_{\perp}\right| \phi(|\lambda|)\right],
$$

where for $|\lambda|=0,1,2, \ldots, \mathcal{N}-1$,

$$
\phi(|\lambda|)=\frac{c}{2} \lambda \operatorname{erf}\left(\lambda \frac{c}{2} \sqrt{\eta}\right)+\frac{1}{\sqrt{\pi \eta}} e^{-\left(\lambda \frac{c}{2} \sqrt{\eta}\right)^{2}} .
$$

We recall that $\left|l^{\prime}-l\right| \frac{c}{2} \equiv \lambda \frac{c}{2}$ is the distance between layers $l^{\prime}, l$. Replacing in Eq. $(41) \mathcal{L}\left(|\lambda| ;\left|\vec{q}_{\perp}\right|\right)$ by its value at $\left|\vec{q}_{\perp}\right|=\overrightarrow{0}$, we obtain

$$
\left.\mathcal{C}_{i j}^{\kappa \kappa^{\prime}}\left(l, l^{\prime} \mid \vec{q}_{\perp}, \vec{\tau}=\overrightarrow{0}\right)\right|_{1}=\frac{2 \pi e_{\kappa}^{* \perp} e_{\kappa^{\prime}}^{* \perp}}{v_{2 \mathrm{D}} \sqrt{m_{\kappa}(l) m_{\kappa^{\prime}}\left(l^{\prime}\right)}} \frac{q_{i} q_{j}}{\left|\vec{q}_{\perp}\right|} .
$$

This expression is independent of the distance between layers $l$ and $l^{\prime}$. It agrees (except for the use of effective charges) with our previous result on 2D h-BN, Eq. (9) of Ref. 30, and is nonanalytic since the derivatives with respect to the wave vector components are not defined at the $\Gamma$ point. In contradistinction to the case of the nonregular Coulomb term in 3D h-BN, the rhs of Eq. (44) has the well-defined limit 0 for $\vec{q}_{\perp} \rightarrow \overrightarrow{0}$. Hence there is no splitting of the highest optical phonon frequencies at $\vec{q}_{\perp}=\overrightarrow{0}$, neither in the monolayer nor in a slab with a finite number of layers $\mathcal{N}$. In this point we disagree with the statement made in an ab initio study ${ }^{20}$ that "the phonon dispersion relations of a single sheet of h-BN are very similar to that of bulk h-BN." For that reason, the criticism in Ref. 20 of the results on the monolayer by Miyamoto et al. ${ }^{23}$ who indeed show no LO-TO splitting, is not justified. We recall that the absence of LO-TO splitting for a monolayer of h-BN at $\Gamma$ has been stressed further in various theoretical papers. ${ }^{24,26}$

Similarly one can show that the contributions to the dynamical matrix arising from the term with $\vec{\tau}_{\perp}=\overrightarrow{0}$ in Eq. (34) (out-of-plane shear displacements) have a welldefined limit for $\vec{q}_{\perp}=\overrightarrow{0}$. Hence there is no discontinuity of $\mathrm{ZO}$ phonon frequencies at $\Gamma$, in contradistinction to the $A_{2 u}$ mode in $3 \mathrm{D} \mathrm{h}-\mathrm{BN}$.

Phonon dispersion relations for slabs of $\mathcal{N}$ layers, obtained by solving the secular equation

$$
\left|1 \omega^{2}-\Delta_{\mathcal{N}}\left(\vec{q}_{\perp}\right)\right|=0,
$$

are shown in Fig. 5.

The main difference between the phonon dispersions of the monolayer and the multilayers is the increasing number of optical branches with increasing number of layers. The lowfrequency part of the optical phonon dispersions $\left(<200 \mathrm{~cm}^{-1}\right)$ reflects the rigid-plane shear and compressional motions. This
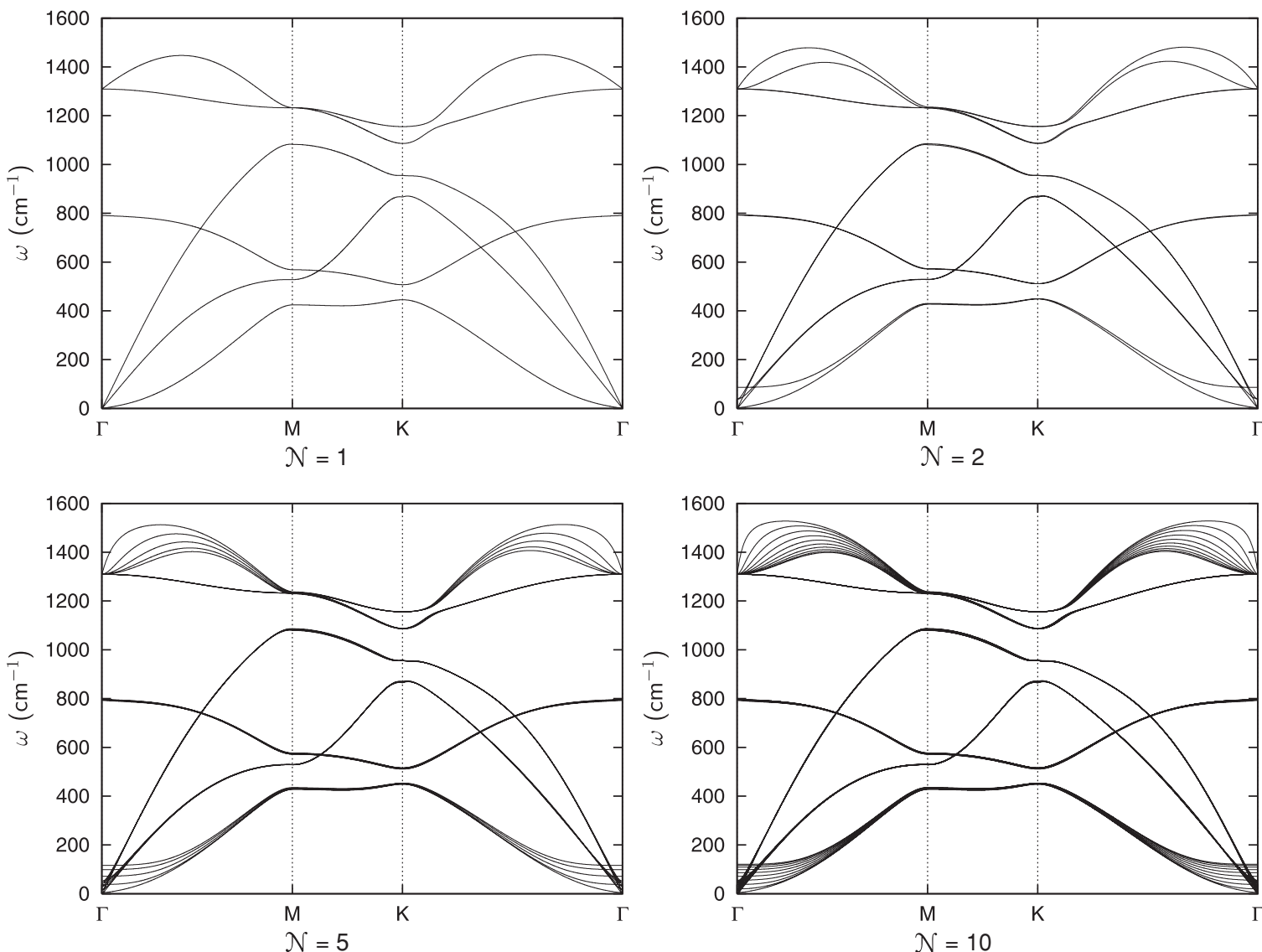

FIG. 5. Phonon dispersions of multilayers, Coulomb forces included. 


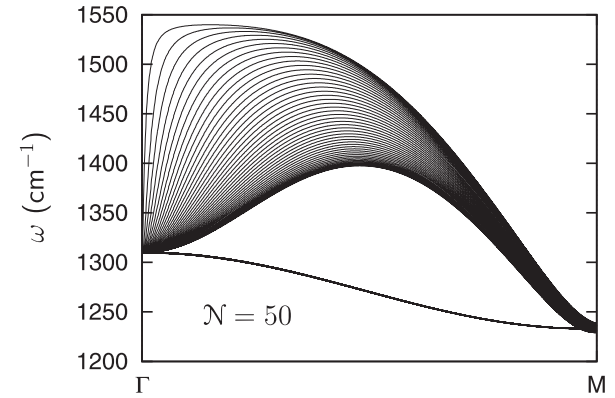

(a)

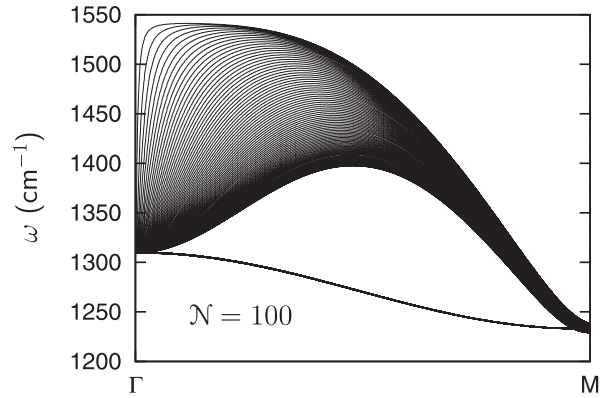

(b) part of the dispersions is very similar to the case of graphene multilayers ${ }^{41}$ where only covalent and van der Waals forces are taken into account. Hence Coulomb forces are rather irrelevant for the rigid-plane optical motion, which is a consequence of the overall charge neutrality of the layers. The optical modes near $800 \mathrm{~cm}^{-1}$ which involve intraplane displacements parallel to the $\vec{c}$ axis remain almost degenerate and independent of the number of layers; here too the Coulomb forces are quasinegligible. On the other hand marked differences between $\mathrm{h}-\mathrm{BN}$ and graphene multilayers appear in the highest optical branches with frequencies $\approx 1300-1550 \mathrm{~cm}^{-1}$. These modes are due to intraplane shear displacements where Coulomb forces are responsible for significant differences. While in the case of graphene multilayers ${ }^{41}$ the highest optical phonon branches are quasidegenerate and coincide with the two highest optical monolayer branches, the degeneracy is lifted for $\vec{q}_{\perp}$ away from $\Gamma$ in h-BN multilayers. For a slab of $\mathcal{N}$ layers a diverging bundle of $\mathcal{N}$ overbending optical phonon branches emerges (Figs. 5 and 6). We recall that overbending means that these optical phonon branches have their maxima away from $\Gamma$ in the 2D BZ. Remarkable also is the increase of the slope of the highest optical branch with increasing number of layers, while the maximum of overbending moves toward the $\Gamma$ point. Thereby this branch approaches more and more the highest branch of 3D h-BN which can be seen as an envelope of the multilayer branches (Fig. 6). An analytical discussion of these results will be given in the next section.

\section{OVERBENDING IN MULTILAYERS}

We will investigate by analytical calculations the $\left|\vec{q}_{\perp}\right|$ dependence of the highest optical phonon frequency for the $\mathcal{N}$-layer system. First, we recall the relevant results for the monolayer case, next we solve the bilayer case, and finally we turn to the $\mathcal{N}$-layer problem.

Previously ${ }^{30}$ we have shown that in $2 \mathrm{D}$ h-BN the highest optical phonon branch which corresponds to intraplane displacements is degenerate at $\Gamma$ with frequency $\omega_{\mathrm{LO}}^{(0)}=\omega_{\mathrm{TO}}^{(0)}$. Away from $\Gamma$, the LO and TO modes are split; for small wave vector $\left|\vec{q}_{\perp}\right|$ the TO frequency is given by $\omega_{\mathrm{TO}}\left(\vec{q}_{\perp}=\overrightarrow{0}\right)=\omega_{\mathrm{TO}}^{(0)}$ while the LO frequency shows a linear increase with $\left|\vec{q}_{\perp}\right|$ :

$$
\omega_{\mathrm{LO}}=\omega_{\mathrm{TO}}^{(0)}+c_{\mathrm{LO}}\left|\vec{q}_{\perp}\right|+\mathcal{O}\left(\left|\vec{q}_{\perp}\right|^{2}\right),
$$

where

$$
c_{\mathrm{LO}}=\frac{\pi\left(e_{\mathrm{B}}^{* \perp}\right)^{2}}{v_{2 \mathrm{D}} \mu \omega_{\mathrm{TO}}^{(0)}} .
$$

(Here we now use the effective charge $e_{\mathrm{B}}^{* \perp}$.)
Studying the dispersions of the highest optical phonons for the bilayer case we proceed by perturbation theory in a similar way as done for the LO-TO splitting in Sec. III. We separate the dynamical matrix $\Delta_{2}\left(\vec{q}_{\perp}\right)$ into an analytic part $\mathcal{R}\left(\vec{q}_{\perp}\right)$ and a nonanalytic part $\left.\mathcal{C}\left(\vec{q}_{\perp}, \vec{\tau}=\overrightarrow{0}\right)\right|_{1}$ :

$$
\Delta_{2}\left(\vec{q}_{\perp}\right)=\mathcal{R}\left(\vec{q}_{\perp}\right)+\left.\mathcal{C}\left(\vec{q}_{\perp}, \vec{\tau}=\overrightarrow{0}\right)\right|_{1} .
$$

We assume that the eigenvalues and eigenvectors of $\mathcal{R}\left(\vec{q}_{\perp}=\overrightarrow{0}\right)$ are known and we will solve the secular problem by considering $\left.\mathcal{C}\left(\vec{q}_{\perp}, \vec{\tau}=\overrightarrow{0}\right)\right|_{1}$ as a perturbation in the long-wavelength regime. Considering only in-plane displacements, $\Delta_{2}\left(\vec{q}_{\perp}\right)$ is of dimension $8 \times 8$. Excluding acoustic phonons (2) and rigid layer optical modes (2), there remain four optical modes corresponding to intraplane displacements. Each displacement vector has 8 components with labels $(l, \kappa, i)$, where $l=0,1$, $\kappa=\mathrm{B}, \mathrm{N}$, and $i=x, y$. The eigenvectors at $\left|\vec{q}_{\perp}\right|=0$ separate in a pair (displacements in the $x$ and $y$ directions, respectively) of uneven (ungerade) modes

$$
\begin{gathered}
\vec{\xi}^{(1)}=\sqrt{\frac{\mu}{2}}\left(\frac{1}{\sqrt{m_{\mathrm{B}}}}, 0,-\frac{1}{\sqrt{m_{\mathrm{N}}}}, 0, \frac{1}{\sqrt{m_{\mathrm{B}}}}, 0,-\frac{1}{\sqrt{m_{\mathrm{N}}}}, 0\right), \\
\vec{\xi}^{(2)}=\sqrt{\frac{\mu}{2}}\left(0, \frac{1}{\sqrt{m_{\mathrm{B}}}}, 0,-\frac{1}{\sqrt{m_{\mathrm{N}}}}, 0, \frac{1}{\sqrt{m_{\mathrm{B}}}}, 0,-\frac{1}{\sqrt{m_{\mathrm{N}}}}\right),
\end{gathered}
$$

and a pair of even (gerade) modes

$$
\begin{aligned}
& \vec{\xi}^{(3)}=\sqrt{\frac{\mu}{2}}\left(\frac{1}{\sqrt{m_{\mathrm{B}}}}, 0,-\frac{1}{\sqrt{m_{\mathrm{N}}}}, 0,-\frac{1}{\sqrt{m_{\mathrm{B}}}}, 0, \frac{1}{\sqrt{m_{\mathrm{N}}}}, 0\right), \\
& \vec{\xi}^{(4)}=\sqrt{\frac{\mu}{2}}\left(0, \frac{1}{\sqrt{m_{\mathrm{B}}}}, 0,-\frac{1}{\sqrt{m_{\mathrm{N}}}}, 0,-\frac{1}{\sqrt{m_{\mathrm{B}}}}, 0, \frac{1}{\sqrt{m_{\mathrm{N}}}}\right) .
\end{aligned}
$$

Transforming to the basis of intraplane optical displacements, we have

$$
\begin{aligned}
\hat{\mathcal{R}}_{v v^{\prime}}\left(\vec{q}_{\perp}=0\right) & \\
\quad= & \sum_{l l^{\prime}} \sum_{\kappa \kappa^{\prime}} \sum_{i j} \xi^{(v)}(l \kappa i) \mathcal{R}_{i j}^{\kappa \kappa^{\prime}}\left(l, l^{\prime} \mid \vec{q} \perp \overrightarrow{0}\right) \xi^{\left(v^{\prime}\right)}\left(l^{\prime} \kappa^{\prime} j\right) \\
& =\delta_{v v^{\prime}}\left(\omega_{p}^{(0)}\right)^{2},
\end{aligned}
$$

where $p$ stands for $u$ if $v\left(v^{\prime}\right) \in\{1,2\}$ and for $g$ if $v\left(v^{\prime}\right)$ $\in\{3,4\}$. Here $\omega_{u}^{(0)}$ and $\omega_{g}^{(0)}$ are the eigenfrequencies of the "unperturbed" modes. Notice that there is complete symmetry 
between the $x$ and $y$ directions as a consequence of the 2D hexagonal structure.

The elements of the matrix $\left.\mathcal{C}\left(\vec{q}_{\perp}, \vec{\tau}=\overrightarrow{0}\right)\right|_{1}$ are specified by Eq. (41), where we replace the function $\mathcal{L}\left(|\lambda| ;\left|\vec{q}_{\perp}\right|\right)$ by its longwavelength approximation [Eqs. (42) and (43)]. Transforming $\left.\mathcal{C}\left(\vec{q}_{\perp}, \vec{\tau}=\overrightarrow{0}\right)\right|_{1}$ to the space of optical modes $\vec{\xi}^{(v)}$ of the twolayer problem, we obtain with $v\left(v^{\prime}\right) \in\{1,2\}$

$$
\left.\hat{\mathrm{C}}\left(\vec{q}_{\perp}, \vec{\tau}=\overrightarrow{0}\right)\right|_{1}=2 \alpha \frac{q_{\nu} q_{\nu^{\prime}}}{\left|\vec{q}_{\perp}\right|}\left\{2-\left|\vec{q}_{\perp}\right|[\phi(|0|)+\phi(|1|)]\right\},
$$

where for $v\left(v^{\prime}\right)=1$ and $2, q_{1}=q_{x}$ and $q_{2}=q_{y}$, respectively. Here we have defined the material constant $\alpha=$ $\pi\left(e_{\mathrm{B}}^{* \perp}\right)^{2} / v_{2 \mathrm{D}} \mu$. With $v\left(v^{\prime}\right) \in\{3,4\}$ we get

$$
\left.\hat{\mathrm{C}}\left(\vec{q}_{\perp}, \vec{\tau}=\overrightarrow{0}\right)\right|_{1}=2 \alpha q_{\nu} q_{\nu^{\prime}}[\phi(|1|)-\phi(|0|)],
$$

where for $v\left(v^{\prime}\right)=3$ and $4, q_{3}=q_{x}$ and $q_{4}=q_{y}$, respectively. Mixed elements between $v \in\{1,2\}$ and $v^{\prime} \in\{3,4\}$ vanish. It then follows that the $4 \times 4$ secular matrix $\left[1 \omega^{2}-\hat{\Delta}_{2}\left(\vec{q}_{\perp}\right)\right]$ becomes quasidiagonal and the secular equation factorizes into two separate determinantal equations:

$$
\left|\left(\omega^{2}-\omega_{p}^{2}\right) \delta_{\nu v^{\prime}}-\hat{\mathcal{C}}_{\nu v^{\prime}}\left(\vec{q}_{\perp}, \vec{\tau}_{\perp}=\overrightarrow{0}\right)\right|_{1} \mid=0,
$$

for $v\left(v^{\prime}\right) \in\{1,2\}, p=u$, and $v\left(v^{\prime}\right) \in\{3,4\}, p=g$. Solving for the case $v\left(v^{\prime}\right) \in\{1,2\}$ we obtain for small $\left|\vec{q}_{\perp}\right|$ the phonon frequencies

$$
\begin{gathered}
\omega_{1}=\omega_{u}^{(0)}, \\
\omega_{2}=\omega_{u}^{(0)}+\frac{2 \alpha}{\omega_{u}^{(0)}}\left|\vec{q}_{\perp}\right|+\mathcal{O}\left(\left|\vec{q}_{\perp}\right|^{2}\right),
\end{gathered}
$$

and for the case $v\left(v^{\prime}\right) \in\{3,4\}$

$$
\begin{gathered}
\omega_{3}=\omega_{g}^{(0)}, \\
\omega_{4}=\omega_{g}^{(0)}+\frac{\alpha q_{\perp}^{2}}{\omega_{g}^{(0)}}[\phi(|1|)-\phi(|0|)] .
\end{gathered}
$$

These four optical phonon branches can be clearly seen in Fig. 7, where we have zoomed in on the numerically obtained phonon dispersions near $\Gamma$ around $\omega=1310 \mathrm{~cm}^{-1}$ (cf. Fig. 5 , $\mathcal{N}=2$ ). We notice that the unperturbed frequencies $\omega_{u}^{(0)}$ and $\omega_{g}^{(0)}$ have almost the same numerical values as the frequency $\omega_{\mathrm{TO}}^{(0)}$ of the monolayer problem. This is a consequence of the

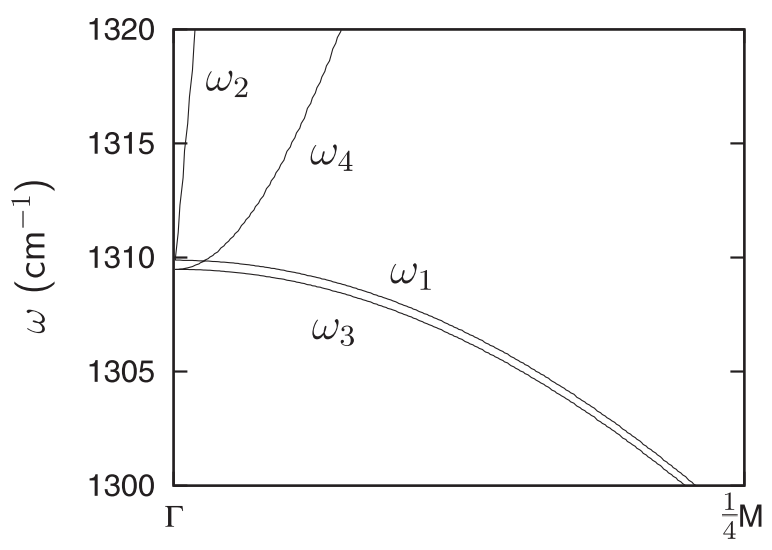

FIG. 7. Detailed highest optical phonon dispersions near $\Gamma$ for the bilayer. weakness of the interlayer forces. Effectively one can assume that $\omega_{1} \approx \omega_{3}$ and in accordance with Fig. 5 (case $\mathcal{N}=2$ ), there are three discernible phonon branches which emerge from $\left|\vec{q}_{\perp}\right|=0$ for the case of two layers. For small wave vectors, the lowest branch has frequency $\omega_{1}$, the intermediate branch has frequency $\omega_{4}$ and increases quadratically with $\left|\vec{q}_{\perp}\right|$, and the highest branch has frequency $\omega_{2}$ and increases linearly with $\left|\vec{q}_{\perp}\right|$. Comparing the slope of this branch with the slope $c_{\mathrm{LO}}$ of the LO monolayer branch, we find $2 \alpha / \omega_{u}^{(0)}=2 c_{\mathrm{LO}}$; i.e., the slope of the highest optical phonon branch of the bilayer is twice the slope of the highest branch of the monolayer.

In the general case of $\mathcal{N}$ layers, there are $2 \mathcal{N}$ optical modes due to intraplane displacements. Near $\Gamma, \mathcal{N}$ of these modes are quasidegenerate (indiscernible in Figs. 5 and 6) while the $\mathcal{N}$ remaining modes show a marked overbending. While a full analytical treatment for the case of $\mathcal{N}$ layers is out of order, a discussion of the highest optical phonon branch is still tractable. Each displacement vector has $4 \mathcal{N}$ components $(l, \kappa, i)$ where $l=0,1,2, \ldots, \mathcal{N}-1, \kappa=\mathrm{B}, \mathrm{N}$, and $i=x, y$. We take advantage of the fact that the $2 \mathcal{N} \times 2 \mathcal{N}$ secular matrix is again quasidiagonal with $2 \times 2$ submatrices. We restrict ourselves to the $2 \times 2$ secular determinant which yields the largest eigenvalue. At $\left|\vec{q}_{\perp}\right|=\overrightarrow{0}$, the corresponding pair of uneven eigenvectors can be explicitly written down:

$$
\begin{aligned}
& \vec{\xi}^{(1)}=\sqrt{\frac{\mu}{\mathcal{N}}}\left(\frac{1}{\sqrt{m_{\mathrm{B}}}}, 0,-\frac{1}{\sqrt{m_{\mathrm{N}}}}, 0, \ldots, \frac{1}{\sqrt{m_{\mathrm{B}}}}, 0,-\frac{1}{\sqrt{m_{\mathrm{N}}}}, 0\right), \\
& \vec{\xi}^{(2)}=\sqrt{\frac{\mu}{\mathcal{N}}}\left(0, \frac{1}{\sqrt{m_{\mathrm{B}}}}, 0,-\frac{1}{\sqrt{m_{\mathrm{N}}}}, \ldots, 0, \frac{1}{\sqrt{m_{\mathrm{B}}}}, 0,-\frac{1}{\sqrt{m_{\mathrm{N}}}}\right) .
\end{aligned}
$$

Denoting the degenerate eigenvalue of the $2 \times 2$ matrix $\hat{\mathcal{R}}\left(\vec{q}_{\perp}=\overrightarrow{0}\right)$ by $\left(\omega_{u}^{(0)}\right)^{2}$, and calculating the elements of the perturbation matrix

$\left.\hat{\mathrm{C}}_{\nu \nu^{\prime}}\left(\vec{q}_{\perp}, \vec{\tau}_{\perp}=\overrightarrow{0}\right)\right|_{1}=2 \alpha \frac{q_{\nu} q_{\nu^{\prime}}}{\left|\vec{q}_{\perp}\right|}\left\{\mathcal{N}-\left|\vec{q}_{\perp}\right| \sum_{\lambda=0}^{\mathcal{N}-1} \phi(|\lambda|)\right\}$,

we obtain by solving the $2 \times 2$ secular determinant the eigenfrequencies

$$
\begin{gathered}
\omega_{1}=\omega_{u}^{(0)}, \\
\omega_{2}=\omega_{u}^{(0)}+\frac{\alpha \mathcal{N}}{\omega_{u}^{(0)}}\left|\vec{q}_{\perp}\right|+\mathcal{O}\left(\left|\vec{q}_{\perp}\right|^{2}\right) .
\end{gathered}
$$

We conclude that for a slab of $\mathcal{N}$ layers, the slope of the largest optical phonon frequency near $\Gamma$ increases proportionally with $\mathcal{N}$. Given the successful experimental measurements of overbending by resonant Raman scattering ${ }^{27}$ in $3 \mathrm{D}$ h-BN, this technique is probably the most appropriate method to check our theoretical results on $\mathrm{h}-\mathrm{BN}$ multilayers.

\section{PIEZOELECTRICITY}

An ionic crystal is piezoelectric if it has no center of symmetry. ${ }^{44}$ Since $3 \mathrm{D}$ h-BN has point group symmetry $D_{6 h}$ it is not piezoelectric. On the other hand $2 \mathrm{D} h-\mathrm{BN}$ with point group symmetry $D_{3 h}$ is the structurally most simple crystal which is piezoelectric. ${ }^{30}$ In the following we will investigate 
the piezoelectricity of h-BN multilayer crystals as a function of the number $\mathcal{N}$ of layers. We will use analytical methods and extend Born's long-wave theory, originally developed for 3D crystals, ${ }^{31,32}$ to the case of multilayer crystals.

Within Born's theory the piezoelectric stress constants $e_{i, j k}$ for 3D crystals are obtained by lattice dynamics:

$$
e_{i, j l} \equiv[i, j l]=\frac{1}{v_{3 \mathrm{D}}} \sum_{\kappa \kappa^{\prime} \kappa^{\prime \prime}} \sum_{h} \sqrt{m_{\kappa}} D_{j h, l}^{\kappa \kappa^{\prime}(1)} \Gamma_{h i}^{\kappa \kappa^{\prime \prime}} \frac{e_{\kappa^{\prime \prime}}^{*}}{\sqrt{m_{\kappa^{\prime \prime}}}} .
$$

Here $v_{3 \mathrm{D}}$ is the volume of the $3 \mathrm{D}$ unit cell, $D_{j h, l}^{\kappa \kappa^{\prime}(1)}$ is the first-order expansion coefficient of the dynamical matrix with respect to the wave vector, $\Gamma_{h i}^{\kappa \kappa^{\prime \prime}}$ is the inverse of the optical part of the dynamical matrix, and $e_{\kappa^{\prime \prime}}^{*}$ is the effective ionic charge. One sees that $e_{i, j l}$ has the dimension of $\mathrm{C} / \mathrm{cm}^{2}$ (C stands for Coulomb), in agreement with the macroscopic theory where $e_{i, j l}$ is defined as the derivative of the polarization (per unit volume) with respect to strain. Evaluating the right-hand side of Eq. (60) for the case of 3D h-BN we obtain zero, a consequence of the fact that the crystal is centrosymmetric. On the other hand, in $2 \mathrm{D}$ crystals the piezoelectric stress is defined as the derivative of the polarization per unit area with respect to strain and has dimension $\mathrm{C} / \mathrm{cm}$. The lattice dynamical expression reads ${ }^{30}$

$$
e_{i, j l} \equiv[i, j l]=\frac{1}{v_{2 \mathrm{D}}} \sum_{\kappa \kappa^{\prime} \kappa^{\prime \prime}} \sum_{h} \sqrt{m_{\kappa}} D_{j h, l}^{\kappa \kappa^{\prime}(1)} \Gamma_{h i}^{\kappa \kappa^{\prime \prime}} \frac{e_{\kappa^{\prime \prime}}^{*}}{\sqrt{m_{\kappa^{\prime \prime}}}} .
$$

Here $v_{2 \mathrm{D}}$ stands for the area of the 2D unit cell; the quantities $D_{j h, l}^{\kappa \kappa^{\prime}(1)}$ and $\Gamma_{h i}^{\kappa \kappa^{\prime \prime}}$ are those of the 2D crystal. In the case of $2 \mathrm{D}$ h-BN there exists only one independent piezoelectric stress constant $e_{1,11}$. Using the valence force constants of Ref. 30 and the charges $e_{\mathrm{B}}^{\perp}=-e_{\mathrm{N}}^{\perp}=0.56 e$ for the calculation of $D_{11,1}^{B N(1)}$ and the effective charge $e_{\mathrm{B}}^{* \perp}=1.218 e$ to account for the long-wavelength displacements, we obtain $\left.e_{1,11}\right|_{2 \mathrm{D}}=$ $2.4340 \times 10^{-12} \mathrm{C} / \mathrm{cm}$. We notice that this value differs from $\left.e_{1,11}\right|_{2 \mathrm{D}}=-1.19 \times 10^{-12} \mathrm{C} / \mathrm{cm}$ in Ref. 30 since there we have used different charges and a charge transfer of different sign.

Turning to the multilayer case, we start from the dynamical matrix $\Delta_{\mathcal{N}}\left(\vec{q}_{\perp}\right)$, Eq. (26), and separate off the nonanalytic Coulomb term by writing the elements (intra- and inter-plane) as

$$
\mathcal{D}_{i j}^{\kappa \kappa^{\prime}}\left(l, l^{\prime} \mid \vec{q}_{\perp}\right)=\mathcal{R}_{i j}^{\kappa \kappa^{\prime}}\left(l, l^{\prime} \mid \vec{q}_{\perp}\right)+\left.\mathcal{C}_{i j}^{\kappa \kappa^{\prime}}\left(l, l^{\prime} \mid \vec{q}_{\perp}, \vec{\tau}_{\perp}=\overrightarrow{0}\right)\right|_{1} .
$$

Here the "short-range" part $\mathcal{R}$ includes the covalent, the van der Waals, and the remaining Coulomb contributions [compare with Eq. (11)]. We expand $\mathcal{R}$ in powers of small $\vec{q}_{\perp}$ :

$$
\begin{aligned}
\mathcal{R}_{i j}^{\kappa \kappa^{\prime}}\left(l, l^{\prime} \mid \vec{q}_{\perp}\right)= & \mathcal{R}_{i i}^{\kappa \kappa^{\prime}(0)}\left(l, l^{\prime}\right) \delta_{i j} \\
& +i \sum_{k} \mathcal{R}_{i j, k}^{\kappa \kappa^{\prime}(1)}\left(l, l^{\prime}\right) q_{k}+\mathcal{O}\left(q_{\perp}^{2}\right),
\end{aligned}
$$

where the indices take the values $k \in\{x, y\}, i(j) \in\{x, y, z\}$. The first term $\mathcal{R}^{(0)}$ on the rhs is a representative element of the dynamical matrix at the $\Gamma$ point of the $2 \mathrm{D}$ BZ. Solution of the corresponding $6 \mathcal{N} \times 6 \mathcal{N}$ secular equation

$$
\left|1 \omega^{2}-\mathcal{R}_{\mathcal{N}}^{(0)}\left(\vec{q}_{\perp}=\overrightarrow{0}\right)\right|=0
$$

yields the spectrum of optical phonons at $\vec{q}_{\perp}=\overrightarrow{0}$ with nonzero frequencies $\left\{\omega_{\nu} ; \nu=1,2,3, \ldots, 6 \mathcal{N}-3\right\}$ and corresponding
$6 \mathcal{N}$-dimensional eigenvectors with components $\xi^{(v)}\left(l_{\kappa} i\right)$. The elements $\mathcal{R}_{i j, k}^{\kappa \kappa^{\prime}(1)}\left(l, l^{\prime}\right)$ are different from zero since in the hexagonal layer structure the atomic positions $\vec{X}^{\kappa}\left(\vec{n}_{\perp}, l\right)$ are not centers of symmetry. The coefficients $\mathcal{R}_{i j, k}^{\kappa \kappa^{\prime}(1)}\left(l, l^{\prime}\right)$ account for changes of the lattice energy due to relative shifts between different sublattices (also called inner displacements ${ }^{31}$ ). From the hermiticity of the matrix $\Delta_{\mathcal{N}}\left(\vec{q}_{\perp}\right)$ it follows that the elements $\mathcal{R}^{(1)}$ are antisymmetric in the interchange of sublattice indices $(\kappa, l) \leftrightarrow\left(\kappa^{\prime}, l^{\prime}\right)$ :

$$
\mathcal{R}_{i j, k}^{\kappa \kappa^{\prime}(1)}\left(l, l^{\prime}\right)=-\mathcal{R}_{i j, k}^{\kappa^{\prime} \kappa(1)}\left(l^{\prime}, l\right) .
$$

In ionic crystals the matrix $\mathcal{R}_{\ldots, k}^{(1)}$ governs the coupling between optical and accoustic phonons or equivalently between electric and mechanical properties (for the $2 \mathrm{D}$ h-BN, see Ref. 30). If in addition the crystal is noncentrosymmetric, this coupling leads to piezoelectricity. Extending Born's long-wave theory to the case of multilayer slabs, we obtain for the piezoelectric stress constant of the $\mathcal{N}$-layer crystal

$$
e_{i, j k}(\mathcal{N})=\frac{1}{v_{\mathcal{N}}} \delta_{i, j k}(\mathcal{N})
$$

Here $v_{\mathcal{N}}=\mathcal{N} a^{2} c \sqrt{3} / 4=\mathcal{N} v_{1}$ is the volume of the prismatic unit cell (see Fig. 4) with $v_{1}=v_{3 \mathrm{D}} / 2=18.089 \times 10^{-24} \mathrm{~cm}^{3}$ the effective volume per pair of particles. The third rank tensor $\mathcal{S}(\mathcal{N})$ is defined by

$$
\begin{aligned}
\mathcal{S}_{i, j k}(\mathcal{N})= & \sum_{l, l^{\prime}, l^{\prime \prime}} \sum_{\kappa, \kappa^{\prime}, \kappa^{\prime \prime}} \sum_{h} \sqrt{m_{\kappa}(l)} \mathcal{R}_{j h, k}^{\kappa \kappa^{\prime}(1)}\left(l, l^{\prime}\right) \Gamma_{h i}^{\kappa^{\prime} \kappa^{\prime \prime}}\left(l^{\prime}, l^{\prime \prime}\right) \\
& \times \frac{e^{*}\left(l^{\prime \prime} \kappa^{\prime \prime} i\right)}{\sqrt{m_{\kappa^{\prime \prime}\left(l^{\prime \prime}\right)}}} .
\end{aligned}
$$

The summations run over the sublattice indices $l, l^{\prime}, l^{\prime \prime} \in$ $\{0,1, \ldots, \mathcal{N}-1\}$ and $\kappa, \kappa^{\prime}, \kappa^{\prime \prime} \in\{\mathrm{B}, \mathrm{N}\}$. We use effective charges $e^{*}\left(l^{\prime \prime} \kappa^{\prime \prime} i\right)$ which correspond to $e_{\mathrm{B}}^{* \perp}=-e_{\mathrm{N}}^{* \perp}$ for in-plane displacements $(i=x, y)$ and to $e_{\mathrm{B}}^{* \|}=-e_{\mathrm{N}}^{* \|}$ for outof-plane displacements $(i=z)$. The $6 \mathcal{N} \times 6 \mathcal{N}$ matrix $\Gamma$ has the elements

$$
\Gamma_{h i}^{\kappa \kappa^{\prime}}\left(l, l^{\prime}\right)=\sum_{\nu} \frac{\xi^{(v)}(l \kappa i) \xi^{(\nu)}\left(l^{\prime} \kappa^{\prime} i\right)}{\omega_{v}^{2}} \delta_{h i}
$$

due to hexagonal symmetry it is diagonal in the displacement indices $h, i$.

For a slab with an uneven number of layers, the crystal class is $D_{3 h}$ (no center of symmetry). There is one independent piezoelectric coefficient

$$
e_{1,11}(\mathcal{N})=\frac{1}{v_{\mathcal{N}}} S_{1,11}(\mathcal{N}),
$$

while symmetry implies ${ }^{44,45} e_{1,22}=e_{2,12}=-e_{1,11}$. On the other hand, for a slab with an even number of layers the crystal class is $D_{6 h}$ (centrosymmetric) and there is no piezoelectricity. In carrying out numerical calculations, we have used the same potential parameters and charges as specified in Secs. II and III. Starting from the dynamical matrix $\Delta_{\mathcal{N}}\left(\vec{q}_{\perp}\right)$ and using Eqs. (63)-(69) we have calculated the quantity $\mathcal{S}_{1,11}(\mathcal{N})$ for $\mathcal{N} \equiv \mathcal{N}_{u}=1,3,5,7,9$ and obtain the same number, $13.2233 \times 10^{-28} \mathrm{C} \mathrm{cm}$, precise to up to five digits. On the other hand, repeating the calculation for $\mathcal{N} \equiv \mathcal{N}_{g}=2,4,6,8,10$, we 


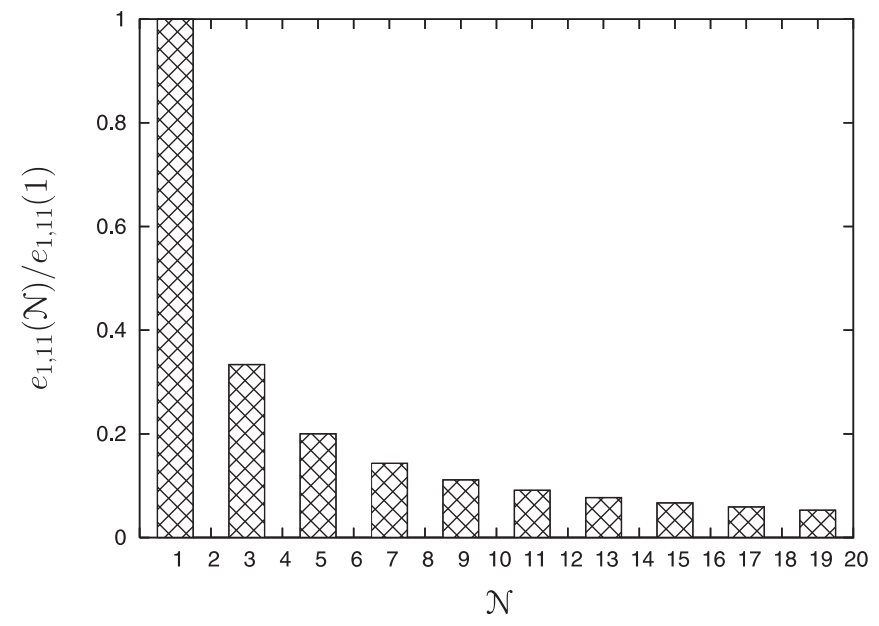

FIG. 8. Fractional change of $e_{1,11}$ with number of layers $\mathcal{N}$.

obtain zero. These results are a consequence of the layered crystal structure with opposite charges alternating along the $\vec{c}$ direction (see Fig. 4 for $\mathcal{N}=3$ ). The symmetry relations Eq. (65) then lead to a cancellation of contributions to $S_{1,11}(\mathcal{N})$ from pairs of layers in the multilayer crystal. Taking into account Eq. (69) and using $v_{\mathcal{N}}=\mathcal{N} v_{1}$ we conclude that the piezoelectric coefficient for slabs with an uneven number of layers $\mathcal{N}_{u}$ equals $e_{1,11}\left(\mathcal{N}_{u}\right)=7.31 \times 10^{-5} \mathrm{C} \mathrm{cm}^{-2} / \mathcal{N}_{u}$, i.e., is inversely proportional to $\mathcal{N}_{u}$. For the case of an even number of layers $\mathcal{N}_{g}, e_{1,11}\left(\mathcal{N}_{g}\right)=0$. These results are illustrated in Fig. 8.

In order to compare this result with the monolayer case where we have obtained $\left.e_{1,11}\right|_{2 \mathrm{D}}=2.4340 \times 10^{-12} \mathrm{C} / \mathrm{cm}$, we assign to the monolayer an effective thickness of $d=c / 2=$ $3.33 \AA$. Thereby we take into account that the $\pi$ electrons extend by $c / 4$ above and below the monolayer plane. We obtain for $\left.e_{1,11}\right|_{2 \mathrm{D}} \times d^{-1}$ the value $7.31 \times 10^{-5} \mathrm{C} / \mathrm{cm}^{2}$ in agreement with $e_{1,11}\left(\mathcal{N}_{u}=1\right)$.

Piezo- and pyroelectric effects have been studied in $\mathrm{BN}$ nanotubes. ${ }^{46,47}$ In particular the axial piezoelectric stress constant in bundles of zig-zag BN nanotubes has been calculated by ab initio methods. The obtained values in Ref. 42 are in the range of $3.89 \times 10^{-5} \mathrm{C} / \mathrm{cm}^{2}$ to $1.86 \times 10^{-5} \mathrm{C} / \mathrm{cm}^{2}$ for $(n, 0)$ nanotubes with $n=5,6, \ldots, 13$; i.e., the axial piezoelectric coefficient decreases with the diameter of the nanotubes.

Even and odd layer effects have been found for other properties in hexagonal multilayer slabs. The unusual flexoelectric effect put forward by first-principles calculations should be present only in thin films of an odd number of h-BN layers. ${ }^{48}$ Within a tight-binding approach it has been shown that Dirac fermions with a linear dispersion are present in graphene stacks only for an odd number of layers; in the case of an even number of layers only normal fermions with a parabolic energy dispersion occur. ${ }^{49}$

\section{CONCLUDING REMARKS AND DISCUSSION}

We have presented a theoretical study of the lattice dynamics of 3D h-BN and of h-BN multilayers. We have described the covalent part of the dynamical matrix by an empirical force constants model ${ }^{30}$ (intraplane interactions) inspired from earlier work on graphite. ${ }^{40}$ Given the ionic character of h-BN as a III-V compound we have calculated the Coulomb term of the dynamical matrix by means of Ewald's method. In order to account for the deformation of the ionic charge distribution by lattice displacements, we have chosen different effective charges for the short-range and the long-range Coulomb contributions (Sec. II). Van der Waals interplane interactions have been described by a phenomenological potential.

The calculated phonon dispersion relations for $3 \mathrm{D} h-\mathrm{BN}$ are shown in Fig. 3. Without Coulomb forces the phonon dispersions [Fig. 3(a)] closely resemble those of graphite. ${ }^{40,41}$ The Coulomb forces mainly influence the highest frequency optical branches $\left(\gtrsim 1300 \mathrm{~cm}^{-1}\right)$ where in-plane ionic displacements are involved [Fig. 3(b)]. We have shown by analytical means that the LO-TO frequency splitting of the $E_{1 u}$ mode is due to the nonregular Coulomb term of the dynamical matrix. The calculated result $\Delta \omega=232 \mathrm{~cm}^{-1}$ is in close agreement with the values obtained from ab initio calculations ${ }^{20,29,43}$ and infrared experiments. ${ }^{13}$ We have also investigated the discontinuity of the $A_{2 u}$ mode frequency as a function of $\vec{q}$ at $\Gamma$.

The calculated phonon dispersion relations of h-BN multilayers (Figs. 5 and 6) also exhibit striking differences with the corresponding dispersions for graphene multilayers in the high-frequency optical part $\left(\gtrsim 1300 \mathrm{~cm}^{-1}\right)$. Although there is no splitting in the BZ center, a bundle of $\mathcal{N}$ strongly diverging branches with pronounced overbending emerges out of the $\Gamma$ point. We interprete this phenomenon as the precursor of the LO-TO splitting found in 3D h-BN. We have studied by analytical means the wave number dependence of the highest optical phonon branch at long wavelengths. We find a linear increase as a function of $\left|\vec{q}_{\perp}\right|$ with a slope proportional to the number of layers $\mathcal{N}$. With increasing $\mathcal{N}$ the maximum of overbending moves closer to $\vec{q}_{\perp}=\overrightarrow{0}$. To our knowledge there are so far no published experimental data on the phonon dispersions of h-BN multilayers.

In the last part of the paper we have studied piezoelectric effects. Since 3D h-BN (point group $D_{6 h}$ ) is centrosymmetric, the crystal is not piezoelectric. However $2 \mathrm{D} h-\mathrm{BN}$ (point group $D_{3 h}$ ) is piezoelectric. If we attribute to a single layer an effective thickness of $c / 2=3.33 \AA$, we find for the piezoelectric stress coefficient of a single layer $e_{1,11}$ $\left(\mathcal{N}_{u}=1\right)=7.31 \times 10^{-5} \mathrm{C} \mathrm{cm}^{-2}$. We find by group theory and by numerical calculations that piezoelectricity vanishes in multilayers with an even number $\mathcal{N}_{g}$ of layers. On the other hand multilayers with an uneven number of layers $\mathcal{N}_{u}$ are piezoelectric, with coefficient $e_{1,11}\left(\mathcal{N}_{u}\right)=e_{1,11}\left(\mathcal{N}_{u}=1\right) / \mathcal{N}_{u}$. These results are a consequence of the layer structure of the crystal where charges with opposite sign but the same absolute value are alternating along the $\vec{c}$ axis. So far this fractional change of piezoelectricity in multilayers (see Fig. 8), to our knowledge, has not been measured. However with progress in the fabrication of ultrathin sheets ${ }^{10-12}$ with a controlled number of layers, experiments to check the above theoretical results might become within reach.

\section{ACKNOWLEDGMENTS}

Discussions with G. Heger, B. Partoens, and F. M. Peeters are gratefully acknowledged. This work has been supported by the Flemish Science Foundation (FWO-Vl) and the Bijzonder Onderzoeksfonds, Universiteit Antwerpen (BOF-UA). 


\section{APPENDIX}

We have to calculate the 2D Fourier component of the Coulomb interactions force constant $\Phi_{i j}^{\kappa \kappa^{\prime}}\left(\vec{n}_{\perp}, l ; \vec{n}_{\perp}^{\prime}, l^{\prime}\right)$ which is defined by

$$
\Phi_{i j}^{\kappa \kappa^{\prime}}\left(l, l^{\prime} \mid \vec{q}_{\perp}\right)=-e_{\kappa} e_{\kappa}^{\prime} \sum_{\vec{n}_{\perp}^{\prime}}\left[\left(\frac{\partial^{2}}{\partial X_{i} \partial X_{j}} \frac{1}{X} e^{i \vec{q}_{\perp} \cdot \vec{X}}\right)\right],
$$

where

$$
\vec{X}=\vec{X}^{\kappa^{\prime}}\left(\vec{n}_{\perp}^{\prime}, l^{\prime}\right)-\vec{X}^{\kappa}\left(\vec{n}_{\perp}, l\right),
$$

with $X=|\vec{X}|$. Using Ewald's method as given in Ref. 32 we separate the Coulomb potential $\phi_{\kappa \kappa^{\prime}}^{C}(X)=e_{\kappa} e_{\kappa^{\prime}} / X$ into

$$
\phi_{\kappa \kappa^{\prime}}^{C}(X)=\phi_{\kappa \kappa^{\prime}}^{(1)}(X)+\phi_{\kappa \kappa^{\prime}}^{(2)}(X)
$$

Here $\phi_{\kappa \kappa^{\prime}}^{(1)}(X)$ is the potential of a point charge $e_{\kappa}$ at a distance $X$ from a 3D Gaussian charge distribution

$$
\rho(X)=e_{\kappa^{\prime}}\left(\frac{\eta}{\pi}\right)^{(3 / 2)} e^{-\eta X^{2}} .
$$

In $3 \mathrm{D}$ space the Fourier transform reads

$$
\phi_{\kappa \kappa^{\prime}}^{(1)}=\frac{1}{(2 \pi)^{3}} \int d^{3} k e^{-i \vec{k} \cdot \vec{X}} \hat{\phi}_{\kappa \kappa^{\prime}}^{(1)}(k),
$$

and from electrostatics (Poisson's equation) one finds

$$
\hat{\phi}_{\kappa \kappa^{\prime}}^{(1)}(k)=\frac{4 \pi e_{\kappa} e_{\kappa^{\prime}}}{k^{2}} e^{-k^{2} / 4 \eta},
$$

where $k^{2}=k_{x}^{2}+k_{y}^{2}+k_{z}^{2}$. The contribution of $\phi_{\kappa \kappa^{\prime}}^{(1)}(X)$ to expression (A1) is written as

$$
\left.\Phi_{i j}^{\kappa \kappa^{\prime}}\left(l, l^{\prime} \mid \vec{q}_{\perp}\right)\right|_{1}=-\sum_{\vec{n}_{\perp}^{\prime}}\left(\frac{\partial^{2}}{\partial X_{i} \partial X_{j}} \phi_{\kappa \kappa^{\prime}}^{(1)}(X)\right) e^{i \vec{q}_{\perp} \cdot \vec{X}} .
$$

We insert Eqs. (A5) and (A6) into (A7) and use the identity

$$
\sum_{\vec{n}_{\perp}^{\prime}} e^{i\left(\vec{q}_{\perp}-\vec{k}_{\perp}\right) \cdot\left[\vec{X}\left(\vec{n}_{\perp}^{\prime}-\vec{X}\left(\vec{n}_{\perp}\right)\right]\right.}=\frac{(2 \pi)^{2}}{v_{2 \mathrm{D}}} \sum_{\vec{\tau}_{\perp}} \delta\left(\vec{q}_{\perp}-\vec{k}_{\perp}-\vec{\tau}_{\perp}\right)
$$

which follows from the 2D periodicity of the slab. Subsequently we integrate over $d k_{\perp}=d k_{x} d k_{y}$ and obtain for $i, j \in x, y$

$$
\begin{aligned}
\left.\Phi_{i j}^{\kappa \kappa^{\prime}}\left(l, l^{\prime} \mid \vec{q}_{\perp}\right)\right|_{1}= & \frac{2 e_{\kappa} e_{\kappa^{\prime}}}{v_{2 \mathcal{D}}} \sum_{\vec{\tau}_{\perp}}\left(\vec{\tau}_{\perp}-\vec{q}_{\perp}\right)_{i}\left(\vec{\tau}_{\perp}-\vec{q}_{\perp}\right)_{j} \\
& \times e^{\left.i \tau_{\perp} \cdot \vec{r}^{\kappa^{\prime}}\left(l^{\prime}\right)-\vec{r}^{\kappa}(l)\right]} e^{-\left|\vec{\tau}_{\perp}-\vec{q}_{\perp}\right|^{2} / 4 \eta} \\
& \times \int_{-\infty}^{+\infty} d k_{z} \frac{e^{-i k_{z}\left(l^{\prime}-l\right) c / 2} e^{-k_{z}^{2} / 4 \eta}}{\left|\vec{q}_{\perp}-\vec{\tau}_{\perp}\right|^{2}+k_{z}^{2}} .
\end{aligned}
$$

Using the integral

$$
\begin{aligned}
\int_{-\infty}^{+\infty} d t \frac{\cos (b t) e^{-a t^{2}}}{x^{2}+t^{2}}= & \frac{\pi}{2 x} e^{a x^{2}}\left\{e^{x b} \operatorname{erfc}\left(x \sqrt{a}+\frac{b}{2 \sqrt{a}}\right)\right. \\
& \left.+e^{-x b} \operatorname{erfc}\left(x \sqrt{a}-\frac{b}{2 \sqrt{a}}\right)\right\}
\end{aligned}
$$

we obtain the result Eqs. (31) and (32).

The calculation of the contribution $\phi_{\kappa \kappa^{\prime}}^{(2)}(X)$ to expression (A1) follows closely the general theory ${ }^{32}$ with the distance between ions given by Eq. (A2). One obtains the result Eq. (33). Turning now to out-of-plane displacements, $i=j=z$, we obtain instead of Eq. (A9)

$$
\begin{aligned}
\left.\Phi_{z z}^{\kappa \kappa^{\prime}}\left(l, l^{\prime} \mid \vec{q}_{\perp}\right)\right|_{1}= & \frac{2 e_{\kappa} e_{\kappa^{\prime}}}{v_{2 \mathrm{D}}} \sum_{\vec{\tau}_{\perp}} e^{i \tau_{\perp} \cdot\left[\vec{r}^{\kappa^{\prime}}\left(l^{\prime}\right)-\vec{r}^{\kappa}(l)\right]} e^{-\left|\vec{\tau}_{\perp}-\vec{q}_{\perp}\right|^{2} / 4 \eta} \\
& \times \int_{-\infty}^{+\infty} d k_{z} \frac{k_{z}^{2} e^{-i k_{z}\left(l^{\prime}-l\right) c / 2} e^{-k_{z}^{2} / 4 \eta}}{\left|\vec{q}_{\perp}-\vec{\tau}_{\perp}\right|^{2}+k_{z}^{2}}
\end{aligned}
$$

Carrying out the integration, we obtain Eq. (33). The contribution $\left.\Phi_{z z}^{\kappa \kappa^{\prime}}\left(l, l^{\prime} \mid \vec{q}_{\perp}\right)\right|_{2}$, Eq. (35), follows again from the general theory.

Finally we investigate the terms $\left.\Phi_{i z}^{\kappa \kappa^{\prime}}\left(l, l^{\prime} \mid \vec{q}_{\perp}\right)\right|_{1,2}$ where $i \in$ $\{x, y\}$. Starting from Eq. (A7) with $j=z$ and $i \neq z$, we obtain by means of Eqs. (A5), (A6), and (A8)

$$
\begin{aligned}
\left.\Phi_{i z}^{\kappa \kappa^{\prime}}\left(l, l^{\prime} \mid \vec{q}_{\perp}\right)\right|_{1} \\
=\frac{2 e_{\kappa} e_{\kappa^{\prime}}}{v_{2 \mathrm{D}}} \sum_{\vec{\tau}_{\perp}}\left(\vec{\tau}_{\perp}-\vec{q}_{\perp}\right)_{i} e^{i \tau_{\perp} \cdot\left[\vec{r}^{\kappa^{\prime}}\left(l^{\prime}\right)-\vec{r}^{k}(l)\right]} e^{-\left|\vec{\tau}_{\perp}-\vec{q}_{\perp}\right|^{2} / 4 \eta} \\
\quad \times \int_{-\infty}^{+\infty} d k_{z} \frac{k_{z} e^{-i k_{z}\left(l^{\prime}-l\right) c / 2} e^{-k_{z}^{2} / 4 \eta}}{\left|\vec{q}_{\perp}-\vec{\tau}_{\perp}\right|^{2}+k_{z}^{2}} .
\end{aligned}
$$

Only the imaginary part of the integral yields a nonzero contribution, with the final result Eqs. (36) and (37).
${ }^{1}$ K. S. Novoselov, A. K. Geim, S. V. Morosov, D. Jiang, Y. Zhang, S. V. Dubonos, I. V. Grigorieva, and A. A. Frisov, Science 306, 666 (2004).

${ }^{2}$ K. S. Novoselov, D. Jian, F. Schedin, T. J. Booth, V. V. Khotkevich, S. V. Morozov, and A. K. Geim, Proc. Nat. Acad. Sci. USA 102, 10451 (2005).

${ }^{3}$ A. K. Geim and K. S. Novoselov, Nature Mater. 6, 183 (2007).

${ }^{4}$ C. Jin, F. Lin, K. Suenaga, and S. Iijima, Phys. Rev. Lett. 102, 195505 (2009).
${ }^{5} \mathrm{~K}$. Watanabe, T. Taniguchi, and H. Kanda, Nature Mater. 3, 404 (2004).

${ }^{6} \mathrm{~T}$. Greber, Graphene and Boron Nitride Layers, in Handbook of Nanophysics, edited by K. Sattler (Taylor and Francis Books, Boca Raton, 2010).

${ }^{7}$ R. Saito, G. Dresselhaus, and M. S. Dresselhaus, Physical Properties of Carbon Nanotubes (Imperial College Press, London, 1998).

${ }^{8}$ A. Loiseau, P. Launois, P. Petit, S. Roche, and J.-P. Salvetat, eds., Understanding Carbon Nanotubes (Springer Verlag, Berlin and Heidelberg, 2006). 
${ }^{9}$ S. Reich, C. Thomsen, and J. Maultzsch, Carbon Nanotubes (Wiley-VCH, Weinheim, 2003).

${ }^{10}$ D. Pacilé, J. C. Meyer, Ç. Ö. Girit, and A. Zettl, Appl. Phys. Lett. 92, 133107 (2008).

${ }^{11}$ W.-Q. Han, L. Wu, Y. Zhu, K. Watanabe, and T. Taniguchi, Appl. Phys. Lett. 93, 223103 (2008).

${ }^{12}$ C. Zhi, Y. Bando, C. Tang, H. Kuwahara, and D. Golberg, Adv. Mater. 21, 2889 (2009)

${ }^{13}$ R. Geick, C. H. Perry, and G. Rupprecht, Phys. Rev. 146, 543 (1966).

${ }^{14}$ R. J. Nemanich, S. A. Solin, and R. M. Martin, Phys. Rev. B 23, 6348 (1981).

${ }^{15}$ T. Kuzuba, K. Era, T. Ishii, and T. Sato, Solid State Commun. 25, 863 (1978).

${ }^{16}$ C. Oshima and A. Nagashima, J. Phys. Condens. Matter 9, 1 (1997).

${ }^{17}$ E. Rokuta, Y. Hasegawa, K. Suzuki, Y. Gamou, C. Oshima, and A. Nagashima, Phys. Rev. Lett. 79, 4609 (1997).

${ }^{18}$ N. G. Chopra, R. J. Luyken, K. Cherrey, V. H. Crespi, M. L. Cohen, S. G. Louie, and A. Zettl, Science 269, 966 (1995).

${ }^{19}$ A. Loiseau, F. Willaime, N. Demoncy, G. Hug, and H. Pascard, Phys. Rev. Lett. 76, 4737 (1996).

${ }^{20}$ G. Kern, G. Kresse, and J. Hafner, Phys. Rev. B 59, 8551 (1999).

${ }^{21}$ N. Ohba, K. Miwa, N. Nagasako, and A. Fukumoto, Phys. Rev. B 63, 115207 (2001).

${ }^{22}$ T. Tohei, A. Kuwabara, F. Oba, and I. Tanaka, Phys. Rev. B 73, 064304 (2006).

${ }^{23}$ Y. Miyamoto, M. L. Cohen, and S. G. Louie, Phys. Rev. B 52, 14971 (1995).

${ }^{24}$ D. Sanchez-Portal and E. Hernandez, Phys. Rev. B 66, 235415 (2002).

${ }^{25}$ W. J. Yu, W. M. Lau, S. P. Chan, Z. F. Liu, and Q. Q. Zheng, Phys. Rev. B 67, 014108 (2003).

${ }^{26}$ L. Wirtz, A. Rubio, R. Arenal de la Concha, and A. Loiseau, Phys. Rev. B 68, 045425 (2003).

${ }^{27}$ S. Reich, A. C. Ferrari, R. Arenal, A. Loiseau, I. Bello, and J. Robertson, Phys. Rev. B 71, 205201 (2005).

${ }^{28}$ A. Bosak, J. Serrano, M. Krisch, K. Watanabe, T. Taniguchi, and H. Kanda, Phys. Rev. B 73, 041402(R) (2006).
${ }^{29}$ J. Serrano, A. Bosak, R. Arenal, M. Krisch, K. Watanabe, T. Taniguchi, H. Kanda, A. Rubio, and L. Wirtz, Phys. Rev. Lett. 98, 095503 (2007).

${ }^{30}$ K. H. Michel and B. Verberck, Phys. Rev. B 80, 224301 (2009).

${ }^{31}$ M. Born and K. Huang, Dynamical Theory of Crystal Lattices (Oxford University Press, Oxford, 1954).

${ }^{32}$ A. A. Maradudin, E. W. Montroll, G. H. Weiss, and I. Ipatova, in Theory of Lattice Dynamics in the Harmonic Approximation, Solid State Physics Suppl. 3, edited by H. Ehrenreich, F. Seitz, and D. Turnbull (Academic Press, New York, 1971).

${ }^{33}$ R. S. Pease, Acta Cryst. 5, 356 (1952).

${ }^{34}$ N. Marom, J. Bernstein, J. Garel, A. Tkatchenko, E. Joselevich, L. Kronik, and O. Hod, Phys. Rev. Lett. 105, 046801 (2010).

${ }^{35}$ M. H. Cohen and F. Keffer, Phys. Rev. 99, 1128 (1955).

${ }^{36}$ R. H. Lyddane and K. F. Herzfeld, Phys. Rev. 54, 846 (1938); R. H. Lyddane, R. G. Sachs, and E. Teller, Phys. Rev. B 59, 673 (1941).

${ }^{37}$ G. B. Grad, P. Blaha, K. Schwarz, W. Auwärter, and T. Greber, Phys. Rev. B 68, 085404 (2003).

${ }^{38}$ A. A. Maradudin, in Dynamical Properties of Solids, edited by G. K. Horton and A. A. Maradudin, Vol. 1 (North-Holland Publishers, Amsterdam, 1974).

${ }^{39}$ X. Gonze and C. Lee, Phys. Rev. B 55, 10355 (1997).

${ }^{40}$ M. Mohr, J. Maultzsch, E. Dobardzic, S. Reich, I. Milosevic, M. Damnjanovic, A. Bosak, M. Krisch, and C. Thomsen, Phys. Rev. B 76, 035439 (2007).

${ }^{41}$ K. H. Michel and B. Verberck, Phys. Rev. B 78, 085424 (2008).

${ }^{42}$ L. A. Girifalco and R. A. Lad, J. Chem. Phys. 25, 693 (1956).

${ }^{43}$ M. Topsakal, E. Aktürk, and S. Ciraci, Phys. Rev. B 79, 115442 (2009).

${ }^{44}$ J. F. Nye, Physical Properties of Crystals (Clarendon Press, Oxford, 1955).

${ }^{45}$ L. D. Landau and E. M. Lifshitz, Elektrodynamik der kontinuierlichen Medien (Akademie Verlag, Berlin, 1987).

${ }^{46}$ E. J. Mele and P. Kral, Phys. Rev. Lett. 88, 056803 (2002).

${ }^{47}$ S. M. Nakhmanson, A. Calzolari, V. Meunier, J. Bernholc, and M. Buongiorno Nardelli, Phys. Rev. B 67, 235406 (2003).

${ }^{48}$ I. Naumov, A. M. Bratkovsky, and V. Ranjan, Phys. Rev. Lett. 102, 217601 (2009).

${ }^{49}$ B. Partoens and F. M. Peeters, Phys. Rev. B 75, 193402 (2007). 